\title{
Multigrid Methods for Helmholtz Problems: A Convergent Scheme in 1D Using Standard Components
}

\author{
Oliver G. Ernst and Martin J. Gander
}

\begin{abstract}
We analyze in detail two-grid methods for solving the 1D Helmholtz equation discretized by a standard finite-difference scheme. We explain why both basic components, smoothing and coarse-grid correction, fail for high wave numbers, and show how these components can be modified to obtain a convergent iteration. We show how the parameters of a two-step Jacobi method can be chosen to yield a stable and convergent smoother for the Helmholtz equation. We also stabilize the coarse-grid correction by using a modified wave number determined by dispersion analysis on the coarse grid. Using these modified components we obtain a convergent multigrid iteration for a large range of wave numbers. We also present a complexity analysis which shows that the work scales favorably with the wave number.
\end{abstract}

Keywords. Multigrid Methods, Helmholtz Equation, 2-step Smoother, Krylov Smoother, Dispersion Correction.

AMS classification. 65N55, 65F10, 65N22.

\section{Introduction}

The development of multigrid methods in the 1960s represents a milestone for solving linear systems of equations obtained by discretizing elliptic PDEs, allowing such a system to be solved with the optimal work and storage complexity, i.e., proportional to the number of unknowns. While enormously effective when applied to elliptic equations as well as a multitude of more difficult problems (see [45] and the references therein), extending the multigrid method for solving the Helmholtz equation with the same efficiency remains an open problem.

The Helmholtz equation

$$
\Delta u+k^{2} u=f
$$

is satisfied by the spatial part $u=u(x)$ of a time-harmonic solution $w(x, t)=$ $u(x) e^{-i \omega t}$ with angular frequency $\omega \in \mathbb{R}$ of the linear wave equation $w_{t t}=c^{2} \Delta w-$ $\tilde{f}$ with propagation speed $c$. For a homogeneous medium, which we shall assume throughout, the wave number $k$ is given by the constant $k=\omega / c$. The wave number is

Please insert information concerning research grant support here. 
real for models of purely propagating waves, which is the case of primary interest and highest difficulty. When the underlying medium is absorbing, $k$ is complex and can be nomalized to have nonnegative real part (cf. [14]).

Multigrid methods consist of an ingeneous combination of the two complementary iterative solution techniques smoothing and coarse-grid correction. Smoothing, in its simplest form, refers to classical so-called relaxation methods, a term introduced by Southwell in the 1930s [43], for successively updating the solution values of a discretized, see also Richardson's early paper [41] from $1910^{1}$. The comprehensive theory of relaxation methods for elliptic PDEs is summarized in the monograph of Varga [49]. Smoothers are characterized algorithmically by updating each solution value by local averages of neighboring values, resulting in linear complexity for each smoothing step. For elliptic PDEs this typically results in strong damping of oscillatory components of the solution error in each step, while smooth components remain essentially unaffected. These smooth error components remaining after smoothing are reduced by the coarse-grid correction, in which an approximation of the original elliptic problem on a coarser grid is solved, in the simplest case by solving the residual equation for the error. This simple combination of smoothing and coarse-grid correction is known as a two-grid iteration; a multigrid method is obtained by solving the coarse grid equation in the same way, descending recursively through increasingly coarser grids and ending ultimately at a grid for which the discrete equation can be solved with trivial effort. Classical multigrid theory is described in [10, 33, 12, 45].

It was realized early on $[4,6,8,42,50]$ that the basic multigrid approach will indeed work for the Helmholtz equation if the wave number is small relative to the mesh size. Essentially, this means that the coarsest grids employed in the multigrid hierarchy must resolve the waves of wave number $k$. This is however not very useful in practical situations, particularly for large-scale calculations, since then the finest grid is precisely chosen to resolve the waves of wave number $k$, and one could not use any coarsening at all in a multigrid context.

Thus, it is still the case that classical multigrid methods do not work when applied to discretizations of the Helmholtz equation, see for example [20, 22] and references therein. Textbooks on multigrid often comment on the difficulty of the case of indefinite problems, for example the early multigrid guide by Brandt and Livne from 1984, which has recently been republished in the SIAM Classics in Applied Mathematics series [10, page 72], contains the quote:

"If $\sigma$ is negative, the situation is much worse, whatever the boundary con-

\footnotetext{
${ }^{1}$ This paper is exemplary for a modern paper in numerical analysis: there are several physical problems modeled by PDEs, which are then discretized, and solved by iteration (Richardson iteration, which is almost the Chebyshev semi-iterative method in its original form, albeit constructing an approximate Chebyshev polynomial by trial and error, and Richardson extrapolation), with realistic wallclock times (flops Richardson and his boy calculators were able to attain), computing costs ( $\frac{n}{18}$ pence per coordinate relaxation of $\Delta_{h}, n$ being the number of digits), and finally there is a real application, a masonry dam.
} 
ditions."

In the formative 1985 monograph by Hackbusch [33, page 212], we find

"We have restricted our interest to weakly indefinite problems since severe difficulties arise for very small $\varepsilon . "$

And in the more recent multigrid book by Trottenberg, Oosterlee and Schüller [45, page 400], the authors state

"The multigrid treatment of the case $c<0$ can become rather involved and will be a real difficulty if $-c$ gets very large."

In these references, the authors then give some brief indications of why standard multigrid methods fail to work effectively for Helmholtz type problems, and many other textbooks simply do not mention the difficult Helmholtz case at all. In the research literature, in an early paper [9], Brandt and Ta'asan investigated why a slight indefiniteness already poses problems for the classical multigrid algorithm, and proposed to treat the small subspace where the algorithm fails explicitly by deflation. This subspace, however, becomes unmanageably large for increasingly large wave number, and therefore one can no longer treat it explicitly when the goal is an algorithm with optimal complexity. A new technique for treating the subspace, namely the ray cycle, was introduced in the PhD thesis of Livshits [39], with a detailed study of the onedimensional case, and a higher-dimensional variant appeared in Brandt and Livshits [11], see also [40]. The so called wave-ray algorithm has, however, a considerable number of technical and algorithmic complications, which make both the implementation and the rigorous convergence analysis challenging, and this has prevented the algorithm from being widely used. A different approach at first sight is to write the Helmholtz equation as a first-order least squares system, see Lee, Manteuffel, McCormick and Ruge [38]. This does, however, in no way remove the specific problems of the multigrid algorithm in the case of the Helmholtz equation, and again ray components need to be introduced, based on several heuristics. While numerical experiments on the unit square illustrate the performance of this method, there is no convergence analysis. Vaněk, Mandel and Brezina present a two-level algebraic multigrid method for the Helmholtz problem in [46], where they use an explicit plane wave representation on the coarse grid, localized within an aggregate of nodes, and zero outside, smoothed by a Chebyshev iteration. Numerical experiments show good computational complexity and scalability of the method. Since multigrid works well in the Helmholtz case provided the mesh is fine enough, and fine meshes are needed because of the pollution effect [35, 36, 3], Kim and Kim propose in [37] to just use standard multigrid for the first few finest levels, and then to switch to a domain decomposition solver of optimized Schwarz type (see below), when multigrid ceases to be effective. Proceeding in a different direction, Elman, Ernst and O'Leary studied the convergence problems of the classical multigrid algorithm applied to the Helmholtz equation in more detail by spectral analysis in [17], and used Krylov methods, both as smoothers and as outer 
convergence accelerator for multigrid, in order to obtain a practical and more robust method. One can also use the Laplacian, as proposed by Bayliss, Goldstein and Turkel [5], or the shifted Laplacian, as advocated by Erlangga, Vuik and Oosterlee [21], as a preconditioner for the Helmholtz equation, and then apply the preconditioner using multigrid, see also [32], and for an algebraic version [2], where it is shown numerically that, asymptotically, the number of iterations grows linearly with the wave number for this approach, see also $[15,48]$. This illustrates well that in the shifted Laplacian preconditioner, there are two conflicting requirements: the shift should be large enough in order for multigrid to work, and not too large, in order to still have a good preconditioner of the original problem, and one cannot, in general, satisfy both; see [22] for a simple Fourier analysis argument, and [31] for a complete theoretical treatment, and the preprint [15] for a detailed local Fourier analysis and a thorough numerical study.

But what happens precisely in the multigrid algorithm when one applies it to the Helmholtz equation? Why and how do standard components of a multigrid algorithm fail? Is it even possible to obtain a convergent algorithm using just the standard components? This chapter is for people who are interested in understanding the details on how multigrid fails for the Helmholtz equation. We explain all our results on a onedimensional model problem, and show what is needed in one dimension in order to obtain a convergent method using only the standard multigrid components of smoothing and coarse-grid correction. Some of the ideas presented in this chapter, such as the smoothing components, can easily be extended to higher dimensions. Others are more difficult to extend, in particular the shifting of the coarse problem, which is a perfect remedy in one spatial dimension, but will not cure all problems of the coarse grid correction in higher dimensions. We hope to share with this text some of our passion we developed over the years analyzing iterative methods for the Helmholtz equation, which has also led to a certain success: for example there are now quite successful incomplete factorization preconditioners, based on the analytic factorization of the operator [26], for the Helmholtz equation see [30] for an analytic incomplete LU (AILU) preconditioner. The newest variants in this class of preconditioners is called the sweeping preconditioner $[18,19]$. Also in domain decomposition there are successful preconditioners, with the first fundamental contribution [16], which then led to optimized Schwarz methods for the Helmholtz equation [29, 28]. There are also specialized FETI methods, like FETI-H [25], and FETI-DPH [23], with a convergence analysis in [24]. For a thorough numerical comparison of all domain decomposition methods specialized for the Helmholtz equation applied to a realistic test equation, see [27]. For multigrid methods, we are certainly still far away from being able to give definitive answers in this chapter, except maybe for one spatial dimension.

The remainder of this chapter is organized as follows: In Section 2 we recall the ineffectiveness and instability of the classical relaxation methods when used as smoothers for the Helmholtz equation using a spectral analysis of the damped Jacobi smoother for a 1D model problem. We propose a two-step Jacobi smoother, a second-order relaxation method, as a possible working alternative and, again using spectral analysis, 
show how its parameters can be chosen depending on the type of indefinitenes, i.e., the level of discretization within a multigrid hierarchy. We also indicate the fundamental limitations of such a stationary iteration at a certain level of the multigrid hierarchy, the so-called resonance level. In Section 3 we perform a detailed spectral analysis of the coarse-grid correction operator using standard linear interpolation and full weighting restriction. We give closed-form expressions of its eigenvalues and show how, with decreasing resolution, the correction operator changes from a projection to an increasingly unstable operation, which can be interpreted as a resonance phenomenon. Guided by dispersion analysis of the finite difference scheme, we explore a variety of modified wave numbers on the coarse grids, resulting in stable correction operators on much coarser grids. Section 4 considers the combination of these remedies in a two-grid iteration and proposes a number of additional modifications yielding, for our model problem, a uniformly convergent two-grid algorithm for essentially all wave numbers that can occur within a full multigrid cycle. Section 5 then gives the results of numerical experiments for solving one-dimensional Helmholtz boundary value problems using multigrid methods constructed from the new techniques.

\section{Smoothing}

Nearly all multigrid algorithms for solving a linear system of equations $\boldsymbol{A} \boldsymbol{u}=\boldsymbol{f}$ employ for their smoothing component stationary linear iterations of the form

$$
\boldsymbol{u}_{m+1}=\boldsymbol{u}_{m}+\boldsymbol{M}^{-1}\left(\boldsymbol{f}-\boldsymbol{A} \boldsymbol{u}_{m}\right), \quad m=0,1, \ldots
$$

based on a splitting $\boldsymbol{A}=\boldsymbol{M}-\boldsymbol{N}$ of the coefficient matrix $\boldsymbol{A}$. Denoting the (algebraic) error of the $m$-th iterate by $\boldsymbol{e}_{m}:=\boldsymbol{u}-\boldsymbol{u}_{m}$ and the associated residual $\boldsymbol{r}_{m}:=\boldsymbol{f}-\boldsymbol{A} \boldsymbol{u}_{m}$, the error propagation operator of such an iteration is given by

$$
\boldsymbol{S}=\boldsymbol{I}-\boldsymbol{M}^{-1} \boldsymbol{A} .
$$

\subsection{Smoothing Analysis}

We give several representations of the spectral properties of smoothers for the 1D model problem

$$
\begin{aligned}
-u^{\prime \prime}(x)-k^{2} u(x) & =f, \quad x \in(0,1), \\
u(0)=u(1) & =0 .
\end{aligned}
$$

With the standard 3-point centered finite difference approximation for the second derivative operator on a uniform mesh with $N$ interior grid points and mesh width $h=1 /(N+1),(2.2)$ is approximated by the linear system $\boldsymbol{A} \boldsymbol{u}=\boldsymbol{f}$ for the function values $u_{j} \approx u\left(x_{j}\right), j=1, \ldots N$, at the grid points $x_{j}=j h$, where

$$
\boldsymbol{A}=\frac{1}{h^{2}} \operatorname{tridiag}\left(-1,2-k^{2} h^{2},-1\right) .
$$



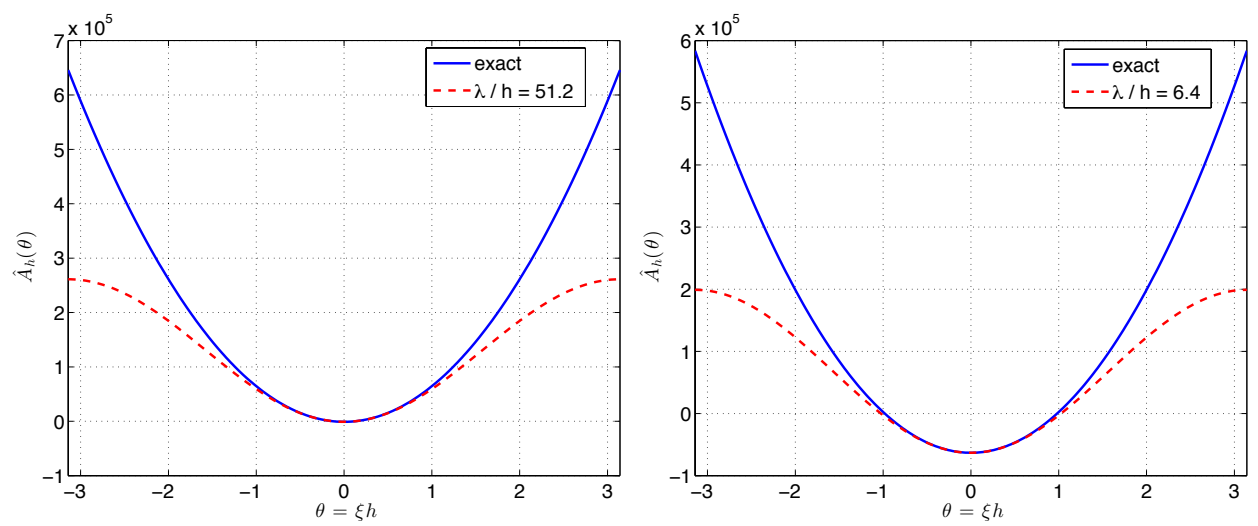

Figure 1. Symbols $\hat{A}(\theta)$ and $\hat{A}_{h}(\theta), \theta=\xi h \in[-\pi, \pi)$ of continuous and discrete 1D Helmholtz operators. The mesh size is $h=1 / 256$ with wave numbers $k_{1}=10 \pi$ and $k_{2}=80 \pi$. The wavelength-to-mesh size ratio $\lambda / h$ is a measure of resolution, indicating how many mesh intervals of length $h$ correspond to each wave of length $\lambda=2 \pi / k$.

\section{Spectral Analysis}

The matrix $\boldsymbol{A}$ is symmetric and therefore has a complete set of orthogonal eigenvectors. These are given by the discrete sine functions

$$
\boldsymbol{v}_{j}=[\sin j \ell \pi h]_{\ell=1}^{N}, \quad j=1, \ldots, N .
$$

When it is necessary to rescale these eigenvectors to have unit Euclidean norm this is achieved by the factor $\sqrt{2 h}$ (for all $j$ ). The associated eigenvalues are given by

$$
\lambda_{j}=\frac{2(1-\cos j \pi h)}{h^{2}}-k^{2}=\frac{4}{h^{2}} \sin ^{2} \frac{j \pi h}{2}-k^{2}, \quad j=1, \ldots, N .
$$

The form of the eigenvectors (2.4) reveals that these become more oscillatory with increasing index $j$. We refer to the eigenpairs accociated with the indices $1 \leq j \leq N / 2$ as the smooth part of the spectrum $I_{\mathrm{sm}}$ and the remainder as the oscillatory part $I_{\mathrm{osc}}$, i.e.,

$$
I_{\mathrm{sm}}:=\left\{\lambda_{j}: 1 \leq j \leq N / 2\right\}, \quad I_{\mathrm{osc}}:=\left\{\lambda_{j}: N / 2<j \leq N\right\} .
$$

When $N$ is odd the eigenpair with index $j=(N+1) / 2$ lies exactly in the middle, and we denote by $n=(N+1) / 2-1=1 /(2 h)-1$, so that the middle index is $n+1$. The associated eigenvector has a wavelength of $4 h$.

\section{Fourier Analysis}

For simple model problems smoothing analysis may be performed based on the spectral decomposition of the error propagation operator (2.1). This usually requires that 
the eigenvalues of $\boldsymbol{S}$ be accessible analytically, as is the case e.g. with Jacobi smoothing applied to Poisson's equation on a square with uniform meshes. An alternative approach, sometimes known as local mode analysis or normal mode analysis, is to perform Fourier analysis, replacing the given boundary conditions by periodic ones or assuming the regular mesh to be infinite. In the latter case the Fourier variable is continuous, in the former discrete. For a given uniform mesh size $h>0$ the infinite grid

$$
x_{j}=j h, \quad j \in \mathbb{Z},
$$

supports Fourier modes

$$
u: \mathbb{Z} \rightarrow \mathbb{C}, \quad u_{j}=e^{i \xi x_{j}}=e^{i \xi j h}, \quad j \in \mathbb{Z},
$$

in which the Fourier variable (frequency) $\xi$ may be restricted to the fundamental domain $\xi \in[-\pi / h, \pi / h)$. In this case, however, the smooth part of the spectrum is given by $I_{\mathrm{sm}}=\left[-\frac{\pi}{2 h}, \frac{\pi}{2 h}\right]$ and the oscillatory part is $I_{\mathrm{osc}}=\left\{|\xi| \geq \frac{\pi}{2 h}\right\}$. Applying the discrete Helmholtz operator $A_{h}=-\Delta_{h}-k^{2}$ resulting from the usual centered three-point finite difference stencil to a Fourier mode (2.7) yields

$$
\begin{aligned}
{\left[\left(-\Delta_{h}-k^{2}\right) u\right]_{j} } & =\frac{1}{h^{2}}\left(-u_{j-1}+2 u_{j}-u_{j+1}\right)-k^{2} u_{j} \\
& =\frac{e^{i \xi x_{j}}}{h^{2}}\left(-e^{-i \xi h}+\left(2-k^{2} h^{2}\right)-e^{i \xi h}\right) \\
& =\frac{e^{i \xi x_{j}}}{h^{2}}\left(2-2 \cos (\xi h)-k^{2} h^{2}\right),
\end{aligned}
$$

which reveals the so-called symbol $\hat{A}_{h}=\hat{A}_{h}(\xi)$ of the discrete Helmholtz operator $A_{h}$ to be

$$
\hat{A}_{h}(\xi)=\frac{2-2 \cos (\xi h)-k^{2} h^{2}}{h^{2}}, \quad \xi \in\left[-\frac{\pi}{h}, \frac{\pi}{h}\right) .
$$

Figure 1 displays the Fourier symbols of the continuous and discrete Helmholtz operators $A=-\Delta-k^{2}$ and $A_{h}=-\Delta_{h}-k^{2}$. To facilitate the comparison of symbols associated with different mesh sizes it is sometimes preferable to plot the symbol against the variable $\theta=\xi h$, which then ranges over the fixed interval $[-\pi, \pi)$.

\subsection{Jacobi Smoothing}

The Jacobi smoother is based on the splitting $\boldsymbol{A}=\boldsymbol{D}-(\boldsymbol{D}-\boldsymbol{A})$, where $\boldsymbol{D}=\operatorname{diag}(\boldsymbol{A})$, resulting in the iteration

$$
\boldsymbol{u}_{m+1}=\boldsymbol{u}_{m}+\boldsymbol{D}^{-1}\left(\boldsymbol{f}-\boldsymbol{A} \boldsymbol{u}_{m}\right)
$$

For smoothing one usually introduces a damping factor $\omega$ for the update, giving

$$
\boldsymbol{u}_{m+1}=\boldsymbol{u}_{m}+\omega \boldsymbol{D}^{-1}\left(\boldsymbol{f}-\boldsymbol{A} \boldsymbol{u}_{m}\right),
$$


which corresponds to the splitting $\boldsymbol{A}=\frac{1}{\omega} \boldsymbol{D}-\left(\frac{1}{\omega} \boldsymbol{D}-\boldsymbol{A}\right)$. The associated error propagation operator is

$$
\boldsymbol{S}_{\omega}=\boldsymbol{I}-\omega \boldsymbol{D}^{-1} \boldsymbol{A} .
$$

Noting that

$$
\boldsymbol{D}=\frac{2-(k h)^{2}}{h^{2}} \boldsymbol{I}
$$

we conclude that $\boldsymbol{A}$ and $\boldsymbol{D}$ are simultaneously diagonalizable, and we obtain for the eigenvalues $\sigma_{j}$ of $\boldsymbol{S}_{\omega}$

$$
\sigma_{j}=\sigma_{j}(\omega)=1-\omega\left(1-\frac{2 \cos (j \pi h)}{2-(k h)^{2}}\right)=: 1-\omega \frac{\lambda_{j}}{\delta}, \quad j=1, \ldots, N,
$$

where we have introduced $\delta=\delta(k, h):=\left(2-k^{2} h^{2}\right) / h^{2}$ to denote the diagonal entry in the Jacobi splitting, which is constant for this model problem. In multigrid methods the smoothing parameter $\omega$ is chosen to maximize damping on the oscillatory half of the spectrum $I_{\text {osc }}$. For the Laplace operator $(k=0)$ the eigenvalues of $\boldsymbol{D}^{-1} \boldsymbol{A}$ are given by $\lambda_{j} / \delta=1-\cos (j \pi h), j=1, \ldots, N$, specifically,

$$
\frac{\lambda_{1}}{\delta}=\frac{\pi^{2} h^{2}}{4}+O\left(h^{4}\right), \quad \frac{\lambda_{N}}{\delta}=2-\frac{\pi^{2} h^{2}}{2}+O\left(h^{4}\right) \quad(h \rightarrow 0) \quad \text { and } \quad \frac{\lambda_{n+1}}{\delta}=1 .
$$

As a result, up to order $h^{2}$, the spectral interval of $\boldsymbol{D}^{-1} \boldsymbol{A}$ is $[0,2]$, with $I_{\text {osc }}=[1,2]$ containing the eigenvalues belonging to oscillatory modes. Maximal damping on $I_{\mathrm{osc}}$ thus translates to the requirement

$$
1-\omega=-(1-2 \omega), \quad \text { i.e., } \quad \omega=\omega_{0}:=\frac{2}{3} .
$$

For this optimal value of the damping parameter $\omega$, each eigenmode belonging to the oscillatory part of the spectrum $\operatorname{span}\left\{\boldsymbol{v}_{n+1}^{h}, \ldots, \boldsymbol{v}_{N}^{h}\right\}$ is reduced by at least a factor of

$$
\sigma_{n+1}\left(\omega_{0}\right)=1-\omega_{0}=\frac{1}{3}
$$

in each smoothing step, independently of the mesh size $h$.

Figure 2 shows the spectrum of $\boldsymbol{S}_{\omega}$ for the discrete 1D Laplacian on the unit interval with mesh width $h=1 / 50$ for the values $\omega=0$ (undamped) and the optimal value $\omega=2 / 3$, on the left plotted against the eigenvalues of $\boldsymbol{A}$. Another common way to visualize the spectral properties of a smoother shown on the right is to plot the eigenvalues of $\boldsymbol{S}_{\omega}$ against the eigenvalue index $j$ or, in a more normalized representation, against $j h$, in which the eigenvalues of the smoother lie on a curved line.

The same considerations applied to the 1D Helmholtz operator $(k>0)$ result in eigenvalues

$$
\frac{\lambda_{j}}{\delta}=1-\frac{2 \cos j \pi h}{2-k^{2} h^{2}}, \quad j=1, \ldots, N,
$$



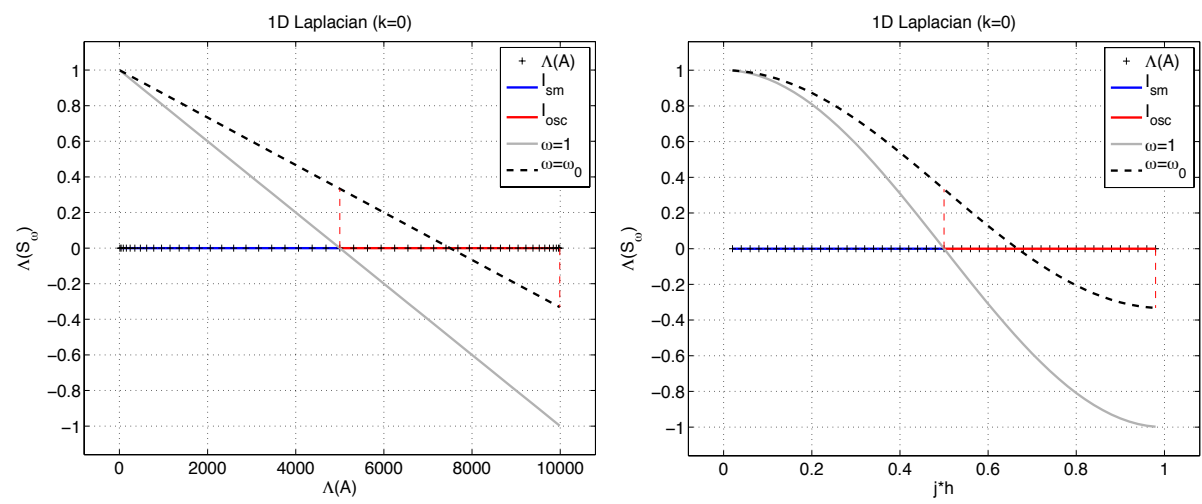

Figure 2. Left: eigenvalues of the undamped and optimally damped Jacobi smoother plotted against those of the associated 1D Laplacian $-\Delta_{h}, h=1 / 50$, divided into smooth and oscillatory parts $I_{\mathrm{sm}}$ and $I_{\mathrm{osc}}$. The dashed red lines indicate the spectral radius of $S_{\omega}$ restricted to the space of oscillatory eigenfunctions. Right: same results, but with smoother eigenvalues $\sigma_{j}$ plotted against $j h$.

of $\boldsymbol{D}^{-1} \boldsymbol{A}$ and therefore, again up to $O\left(h^{2}\right)$, a spectral interval ranging between the extremal eigenvalues

$$
\frac{\lambda_{1}}{\delta}=\frac{-k^{2} h^{2}}{2-k^{2} h^{2}}, \quad \frac{\lambda_{N}}{\delta}=\frac{4-k^{2} h^{2}}{2-k^{2} h^{2}},
$$

so that maximal smoothing on the oscillatory half of the spectrum is obtained for $\omega$ characterized by

$$
1-\omega \frac{\lambda_{N}}{\delta}=-\left(1-\omega \frac{\lambda_{1}+\lambda_{N}}{2 \delta}\right)
$$

which results in

$$
\omega=\omega_{k}:=\frac{2-k^{2} h^{2}}{3-k^{2} h^{2}} .
$$

Figure 3 shows the spectrum of $\boldsymbol{S}_{\omega}$ for the discrete 1D Helmholtz operator on the unit interval with mesh width $h=1 / 50$ for different values of $\omega$, on the left plotted against the eigenvalues of $\boldsymbol{A}$, and on the right against the eigenvalue index $j$.

Remark 2.1. In the Laplace case, the optimal choice of the smoothing parameter $\omega$ guarantees a reduction of the high-frequency error components by a factor of at least $1 / 3$ independently of the mesh size. This is not so in the case of the Helmholtz equation, since there we obtain for the reduction factor

$$
\left|1-\omega_{k} \frac{\lambda_{N}}{\delta}\right|=\left|\sigma_{N}\left(\omega_{k}\right)\right|=\left|\frac{1+2 \cos N \pi h}{3-k^{2} h^{2}}\right| \approx \frac{1}{3-k^{2} h^{2}} .
$$

This reduction factor is only smaller than one if $k h<\sqrt{2}$, which means the resolution $r:=2 \pi /(k h)$, i.e., the ratio of wavelength $2 \pi / k$ to the mesh size $h$, must be larger than 

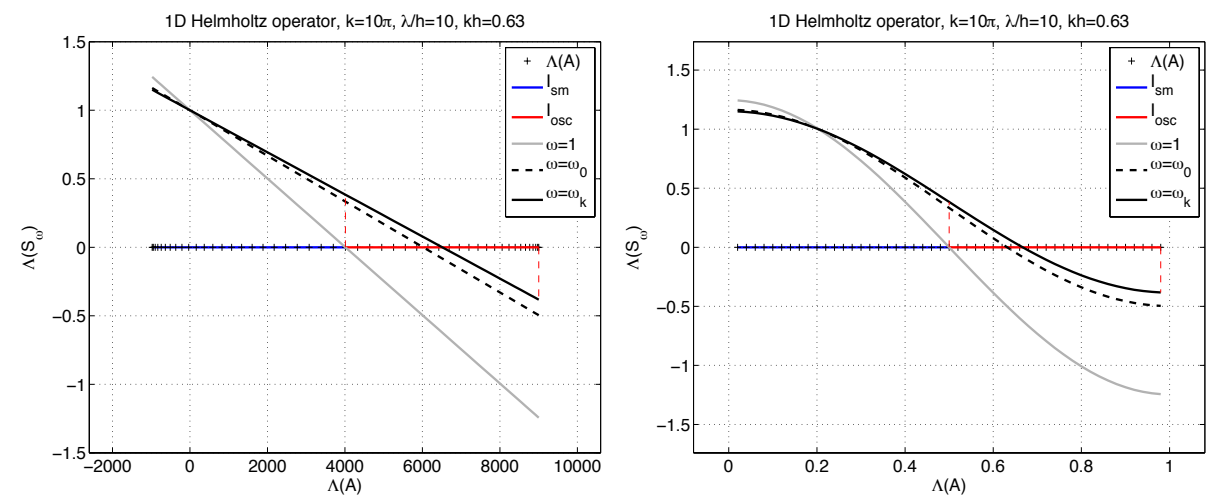

Figure 3. Left: eigenvalues of the Jacobi smoother plotted with $\omega=\omega_{k}, \omega=1$ and $\omega=\omega_{0}$ against those of the associated 1D Helmholtz operator $-\Delta_{h}-k^{2}, h=1 / 50$ with wavelength-to-mesh ratio $\lambda / h=10$. Right: same result in equispaced representation.

$\sqrt{2} \pi \approx 4.44$ points per wavelength, otherwise the smoother is not working. This was also the assumption under which the parameter $\omega_{k}$ was derived. For given $k h<\sqrt{2}$ one has to perform

$$
\nu \geq \frac{-\log \epsilon}{\log \left(3-k^{2} h^{2}\right)}
$$

smoothing steps to achieve the same reduction factor $\epsilon=1 / 3$ as a single smoothing step in the Laplace case. Expanding the number of smoothing steps close to the limit $k h=\sqrt{2}$, we find for the number of smoothing steps the estimate

$$
\nu=\frac{-\sqrt{2} \log \epsilon}{4(\sqrt{2}-k h)}+O(1) .
$$

In addition, the Jacobi smoother is not stable when used for the Helmholtz equation, since low frequency modes are amplified. The strongest amplification occurs for the lowest mode, namely

$$
1-\omega_{k} \frac{\lambda_{1}}{\delta}=\sigma_{1}\left(\omega_{k}\right)=\frac{1+2 \cos \pi h}{3-k^{2} h^{2}} \approx \frac{3}{3-k^{2} h^{2}}>1
$$

for $k h<\sqrt{2}$, and the worst amplification could be as large as 3 .

\subsection{Two-Step Jacobi Smoothing}

As the mesh is coarsened beyond a resolution of roughly $\lambda=8 h$ the standard damped Jacobi smoother becomes less efficient because

(a) there is less damping of modes in the oscillatory part $I_{\mathrm{osc}}$ of the spectrum (cf. $(2.6))$ 

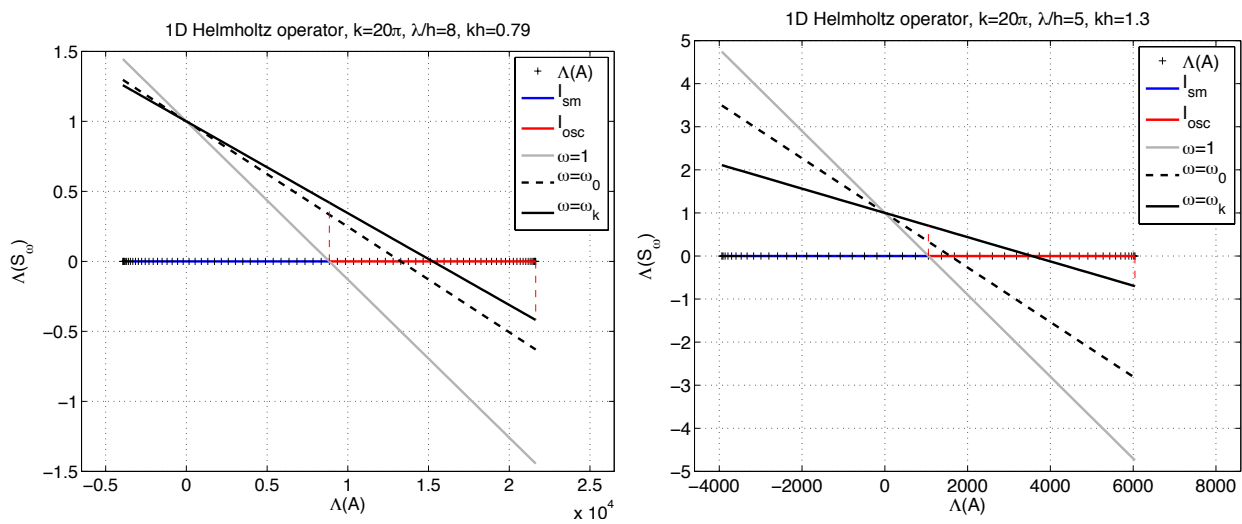

Figure 4. Eigenvalues of the damped Jacobi smoother with larger wavelength-to-mesh ratio $\lambda / h=8$ and $\lambda / h=5$. The damping on $I_{\mathrm{osc}}$ is becoming less while smooth modes at the lower end of $I_{\mathrm{osc}}$ are amplified significantly.

(b) negative eigenvalues in the smooth part $I_{\mathrm{sm}}$ are amplified, some severely.

This is illustrated in Figure 4, where the eigenvalues of the damped Jacobi smoother with different values of the damping parameter are shown for wavelength-to-mesh ratios of 8 and 5. Even for the wavenumber-adapted value $\omega=\omega_{k}$ we observe that damped Jacobi relaxation is becoming less effective as a smoother on $I_{\text {osc }}$ while at the same time amplifying the smoothest modes in $I_{\mathrm{sm}}$, for $\lambda / h=5$ up to a factor of 2 .

More flexibility in the construction of the smoother is obtained for higher-order relaxation methods (cf. [49, Chapter 5]). Rather than allowing arbitrarily high order (cf. the preprint [47] or Krylov smoothers [7, 17]) we consider a two-step Jacobi smoother consisting of two consecutive smoothing steps, each with its own damping parameter $\omega_{1}$ and $\omega_{2}$ :

$$
\boldsymbol{S}_{\omega_{1}, \omega_{2}}=\left(\boldsymbol{I}-\omega_{1} \boldsymbol{D}^{-1} \boldsymbol{A}\right)\left(\boldsymbol{I}-\omega_{2} \boldsymbol{D}^{-1} \boldsymbol{A}\right) .
$$

Such a two-step Jacobi scheme was proposed as a (standalone) solver for Helmholtz problems by Hadley [34].

Denoting again the eigenvalues of $\boldsymbol{D}^{-1} \boldsymbol{A}$ by $\lambda_{j} / \delta$, the eigenvalues $\sigma_{j}$ of $\boldsymbol{S}_{\omega_{1}, \omega_{2}}$ are given by

$$
\sigma_{j}=\sigma\left(\lambda_{j}\right), \quad \sigma(\lambda)=\left(1-\omega_{1} \frac{\lambda}{\delta}\right)\left(1-\omega_{2} \frac{\lambda}{\delta}\right),
$$

i.e., the eigenvalues of the two-step Jacobi smoother lie on a parabola with vertex

$$
\lambda_{v}=\frac{\delta}{2} \frac{\omega_{1}+\omega_{2}}{\omega_{1} \omega_{2}} \quad \text { with value } \quad \sigma\left(\lambda_{v}\right)=-\frac{1}{4} \frac{\left(\omega_{1}-\omega_{2}\right)^{2}}{\omega_{1} \omega_{2}}
$$

To determine values of the smoothing parameters $\omega_{1}$ and $\omega_{2}$ to ensure stability on $I_{\mathrm{sm}}$ as well as good damping on $I_{\mathrm{osc}}$ we distinguish three cases with regard to the location 

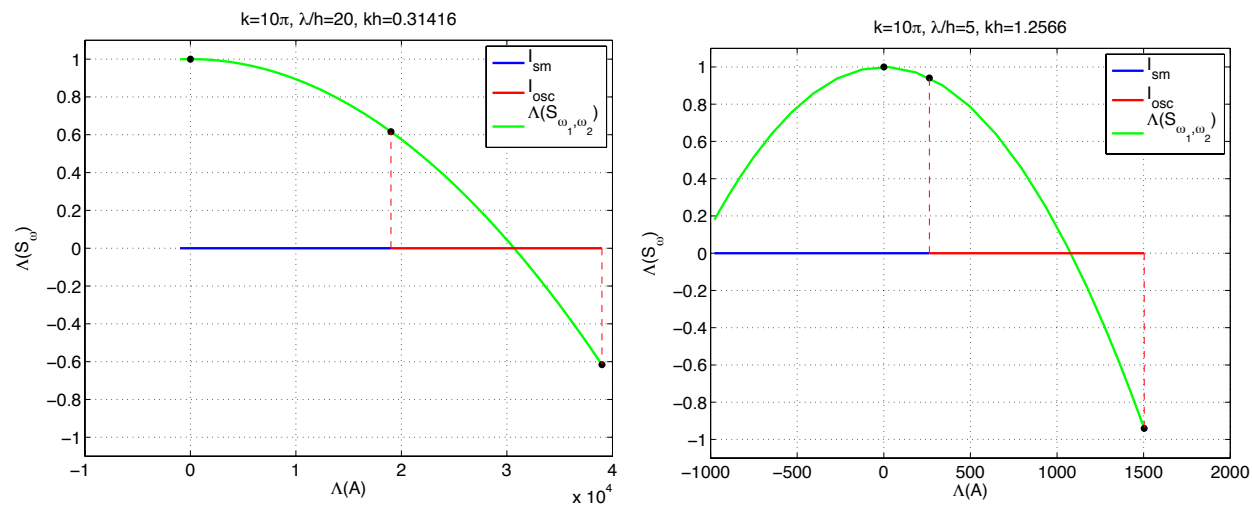

Figure 5. Two-step Jacobi smoothing with parameter choice (2.14).

of the two halves of the spectral interval $\left[\lambda_{1}, \lambda_{N}\right]$ relative to the origin. Specifically, introducing

$$
\lambda_{\text {mid }}:=\frac{\lambda_{1}+\lambda_{N}}{2}
$$

we distinguish as to whether

$$
0<\lambda_{\text {mid }}, \quad \lambda_{\text {mid }}<0<\lambda_{N}, \quad \text { or } \quad \lambda_{N}<0,
$$

i.e., whether $I_{\mathrm{osc}}$ lies to the right, contains, or lies to the left of the origin, respectively. Using the estimates $\lambda_{1} \approx-k^{2}$ and $\lambda_{N} \approx 4 / h^{2}-k^{2}$, these cases are characterized by

$$
k h \in[0, \sqrt{2}), \quad k h \in[\sqrt{2}, 2), \quad k h \in[2, \sqrt{6}), \quad \text { and } \quad k h \geq \sqrt{6} .
$$

\section{Case (1): $0<\lambda_{\text {mid }}$}

When $I_{\mathrm{osc}}$ lies to the right of the origin we design the smoother to damp the modes associated with the eigenvalues there while at the same time not amplifying the smooth modes, i.e., those with eigenvalues in $I_{\mathrm{sm}}$, which lie closer to and on the left of the origin. We accomplish this by fixing the maximum value of the parabola $\sigma=\sigma(\lambda)$ at $\lambda=0$ while requiring maximal damping on the oscillatory interval $I_{\mathrm{osc}}$ :

$$
\begin{aligned}
\sigma^{\prime}(0) & =0, \\
\sigma\left(\lambda_{\text {mid }}\right) & =-\sigma\left(\lambda_{N}\right) .
\end{aligned}
$$

The first constraint yields $\omega_{2}=-\omega_{1}$ which, together with the second, results in (note that $\delta>0$ in this case)

$$
\omega_{1}=\sqrt{\frac{8 \delta^{2}}{\lambda_{1}^{2}+2 \lambda_{1} \lambda_{N}+5 \lambda_{N}^{2}}}=\frac{2 \sqrt{2} \delta}{\sqrt{\left(\lambda_{1}+\lambda_{N}\right)^{2}+4 \lambda_{N}^{2}}} .
$$


Figure 5 illustrates this choice of parameters. On the left we see the eigenvalue plot for the two-step Jacobi smoother with this choice of relaxation parameters for a wellresolved situation $\lambda=20 \mathrm{~h}$. Note that damping on $I_{\mathrm{osc}}$ is reasonable, although weaker than for the one-step Jacobi smoother at this resolution. On the right the same plot for a lower resolution of $\lambda=5 h$. Note that damping has become weaker with decreasing resolution, but there is no amplification.

Remark 2.2. Once more, this smoother does not lead to a fixed mesh-independent reduction factor of the high-frequency modes. Specifically, the reduction factor is

$$
\begin{aligned}
\left|\left(1-\omega_{1} \frac{\lambda_{N}}{\delta}\right)\left(1-\omega_{2} \frac{\lambda_{N}}{\delta}\right)\right| & =\left|1-\omega_{1}^{2} \frac{\lambda_{N}^{2}}{\delta^{2}}\right|=\left|1-\frac{8 \lambda_{N}^{2}}{\left(\lambda_{1}+\lambda_{N}\right)^{2}+4 \lambda_{N}^{2}}\right| \\
& \approx \frac{6-2 k^{2} h^{2}}{10-6 k^{2} h^{2}+k^{4} h^{4}},
\end{aligned}
$$

where we have used the estimates $\lambda_{1} \approx-k^{2}$ and $\lambda_{N} \approx 4 / h^{2}-k^{2}$. This reduction factor is again only strictly smaller than one if $k h<\sqrt{2}$, for which it was constructed. For given $k h<\sqrt{2}$, one now has to perform

$$
\nu \geq \frac{\log \epsilon}{\log \left(\left(6-2 k^{2} h^{2}\right) /\left(10-6 k^{2} h^{2}+k^{4} h^{4}\right)\right)}
$$

smoothing steps to get the same reduction factor $\epsilon=1 / 3$ as one smoothing step for the Laplace case, but this smoother is stable. Expanding the number of smoothing steps close to the critical value $k h=\sqrt{2}$, we find

$$
\nu=\frac{-\log \epsilon}{4(\sqrt{2}-k h)^{2}}+O\left(\frac{1}{k h-\sqrt{2}}\right),
$$

which shows that the price for stability one has to pay is the squaring of the smoothing steps close to the resolution limit, compared to the classical single-step Jacobi method.

Case (2): $\lambda_{\text {mid }}<0<\lambda_{N}$

When the center of the spectral interval moves to the left of the origin, requiring equal damping on different sides of the origin can cause the smoother to become unstable for the smooth modes. This can be seen to occur in Figure 6 on the left for a resolution of $\lambda=4 h$. Instead, we require for this situation $\sigma^{\prime}(0)=0$ as before and, to guarantee stability for the smooth modes, $\sigma\left(\lambda_{1}\right)=-1$. This is achieved for the parameters

$$
\omega_{1}=\frac{\sqrt{2}|\delta|}{\left|\lambda_{1}\right|}, \quad \omega_{2}=-\omega_{1} .
$$

Figure 6 on the right shows the smoother for this parameter choice for the same resolution. The amplification of the smooth modes is seen to have been eliminated. However, we see that no damping occurs for modes associated with eigenvalues near the origin. This is an inherent problem for the indefinite Helmholtz operator. 

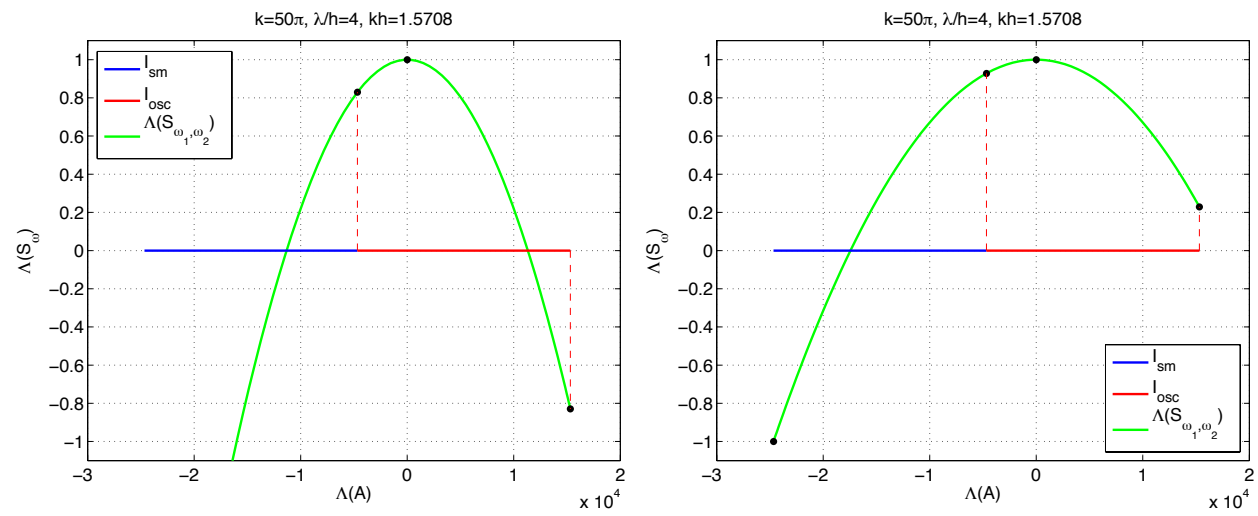

Figure 6. Left: two-step Jacobi smoother with parameter choice (2.14) from Case (1) at resolution $\lambda / h=4$, for which $\lambda_{\text {mid }}<0<\lambda_{N}$. The smooth modes are seen to be strongly amplified. Right: two-step Jacobi smoother with parameter choice (2.16) from Case (2) at resolution $\lambda / h=4$.

Remark 2.3. We call this most difficult level for the smoothing operation the resonance level. The reduction factor we obtain with the two-step Jacobi smoother for each $j=n+1, \ldots, N$ is

$$
\left|\left(1-\omega_{1} \frac{\lambda_{j}}{\delta}\right)\left(1-\omega_{2} \frac{\lambda_{j}}{\delta}\right)\right|=\left|1-\omega_{1}^{2} \frac{\lambda_{j}^{2}}{\delta^{2}}\right|=\left|1-2\left(\frac{4 \sin ^{2} \frac{j \pi h}{2}-k^{2} h^{2}}{4 \sin ^{2} \frac{\pi h}{2}-k^{2} h^{2}}\right)^{2}\right|
$$

This reduction factor can be arbitrarily close to one, since $2 \sin \frac{j \pi h}{2}$ can be arbitrarily close to $k h \in(\sqrt{2}, 2)$ on this level, but they cannot be equal, since otherwise the corresponding Dirchlet problem would be singular. For a reduction by a factor $\epsilon$, one has to perform

$$
\nu \approx \frac{-\log \epsilon}{2}\left[\frac{k^{2} h^{2}}{4 \sin ^{2} \frac{j_{0} \pi h}{2}-k^{2} h^{2}}\right]^{2}
$$

smoothing steps, where $j_{0}$ is the closest integer to the solution of the equation $4 \sin ^{2} \frac{j \pi h}{2}=$ $k^{2} h^{2}$, i.e.

$$
j_{0}=\operatorname{round}\left(\frac{2}{\pi h} \arcsin \frac{k h}{2}\right) .
$$

We arrive at (2.18) by approximating $\log q$, where $q$ denotes the reduction factor in (2.17), to first order by $\log q=\log (1-\delta) \approx-\delta$ for $\delta$ small and bounding $\mid 4 \sin ^{2} \frac{\pi h}{2}-$ $k^{2} h^{2} \mid$ by $k^{2} h^{2}$.

In the best case, $k$ would lie exactly between two adjacent values of $j, k=\frac{4}{h^{2}} \sin ^{2} \frac{\left(j+\frac{1}{2}\right) \pi h}{2}$, and then for a $j>n=\frac{1}{2 h}-1$, e.g. $j=\frac{1}{2 h}+c$ for some integer $c \geq 0$, we obtain for 

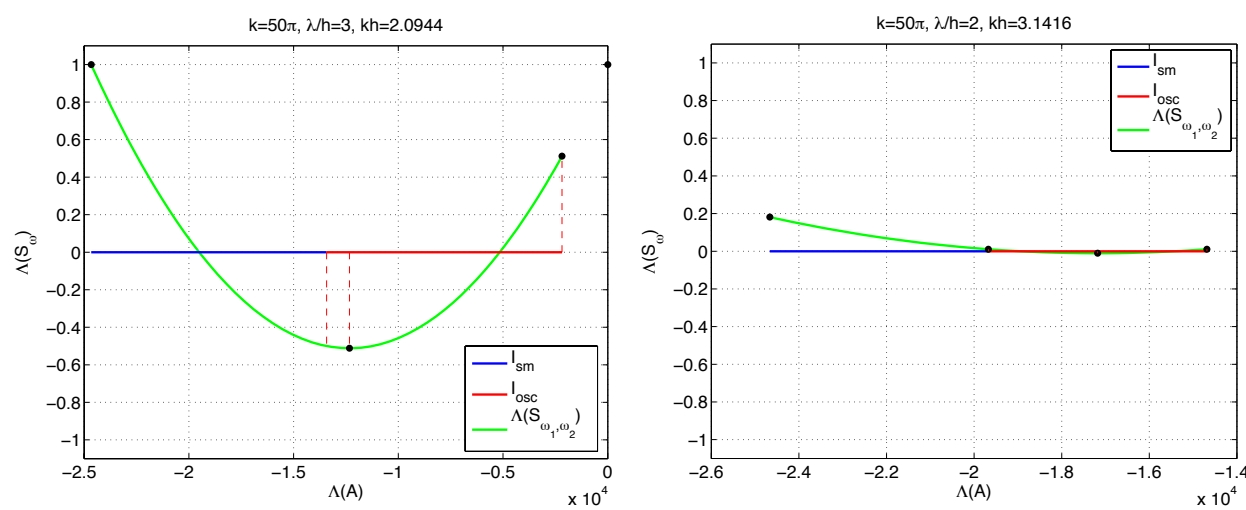

Figure 7. Two-step Jacobi smoothing with parameter choices (2.19) (left) and (2.20) (right), respectively.

$h$ small

$$
\left[4 \sin ^{2} \frac{j \pi h}{2}-k^{2} h^{2}\right]^{2}=\left[4 \sin ^{2} \frac{\left(\frac{1}{2 h}+c\right) \pi h}{2}-4 \sin ^{2} \frac{\left(\frac{1}{2 h}+c+\frac{1}{2}\right) \pi h}{2}\right]^{2}=\pi^{2} h^{2}+O\left(h^{4}\right),
$$

independently of the constant $c$. Since on the resonance level $k h=C$ with $C \in$ $(\sqrt{2}, 2)$, this shows that one has to perform about

$$
\nu \approx-\frac{\log \epsilon}{2} \frac{C^{2} k^{2}}{\pi^{2}}=O\left(k^{2}\right)
$$

smoothing steps for an error reduction by a factor of $\epsilon$ in the high-frequency modes, a truly expensive level for smoothing. It is therefore recommended not to use a simple two-step Jacobi smoother on this level. A better idea would be to use a Chebyshev smoother tuned for the oscillatory part of the spectrum, or even a Krylov method, which can lead to a fixed error reduction with only $O(k)$ iterations.

\section{Case (3): $\lambda_{N}<0$}

In the remaining case where also the largest eigenvalue has crossed to the left, i.e., the problem has become negative definite, we further distinguish between the cases

$$
\lambda_{1}<3 \lambda_{N} \quad \text { and } \quad \lambda_{1}>3 \lambda_{N} .
$$

In terms of the approximations $\lambda_{1} \approx-k^{2}$ and $\lambda_{N} \approx 4 / h^{2}-k^{2}$, this corresponds to whether or not $k h<\sqrt{6}$.

In the first case we fix $\sigma\left(\lambda_{1}\right)=1$, thus making the parabola symmetric to $\lambda=\lambda_{1} / 2$ (since also $\sigma(0)=1$ by construction) and thus ensuring stability. We further specify 
equal damping at the right end of the spectrum $\lambda_{N}$ and at the vertex of the parabola $\lambda_{v}=\lambda_{1} / 2$, i.e., $\sigma\left(\lambda_{v}\right)=-\sigma\left(\lambda_{N}\right)$. This results in

$$
\omega_{1}=\frac{2(2+\sqrt{2}) \delta}{\lambda_{1}+(2+2 \sqrt{2}) \lambda_{N}}, \quad \omega_{2}=\frac{2(2-\sqrt{2}) \delta}{\lambda_{1}+(2-2 \sqrt{2}) \lambda_{N}} .
$$

For the reduction factor on the oscillatory part $I_{\mathrm{osc}}$ of the spectrum we obtain

$$
\left|\sigma\left(\lambda_{N}\right)\right|=\left|\sigma\left(\lambda_{v}\right)\right|=\frac{\left(\lambda_{1}-2 \lambda_{N}\right)^{2}}{\lambda_{1}+4 \lambda_{1} \lambda_{N}-4 \lambda_{N}^{2}}
$$

This situation is depicted on the left of Figure 7.

In the final remaining case $\lambda_{1}>3 \lambda_{N}$, we choose the parabola to be the shifted and scaled Chebyshev polynomial of degree 2 which is 1 at $\lambda=0$ and has minimal supremum on $I_{\mathrm{osc}}$. This is not an option when $\lambda_{1}<3 \lambda_{N}$ since then the Chebyshev polynomial will generally be greater than one on $I_{\mathrm{sm}}$. In this case we obtain the parameters

$$
\omega_{1}=\frac{4(2+\sqrt{2}) \delta}{\lambda_{1}+(7+4 \sqrt{2}) \lambda_{N}}, \quad \omega_{2}=\frac{4(2-\sqrt{2}) \delta}{\lambda_{1}+(7-4 \sqrt{2}) \lambda_{N}} .
$$

This situation at a resolution of $\lambda / h=2$ is shown on the right of Figure 7 . In the limiting case $\lambda_{1}=3 \lambda_{N}$ (2.19) and (2.20) yield the same two parameters.

Remark 2.4. While the smoothing in this last case is again quite effective, there are in general no error components left any more on these levels, if the two-step Jacobi smoother with the $O\left(k^{2}\right)$ smoothing steps required on the previous level was used, as one can see from the error reduction curve shown in Figure 6: with so many smoothing steps, all coarse error components will have been damped out as well, even more so than the oscillatory ones. This may, however, not be so if a special smoother is used on the resonance level.

So for completeness, we give here an estimate of the number of smoothing steps needed. In the first case, $2<k h<\sqrt{6}$, the reduction factor on the high-frequency modes with the parameter values (2.19) is at least

$$
\left|\left(1-\omega_{1} \frac{\lambda_{N}}{\delta}\right)\left(1-\omega_{2} \frac{\lambda_{N}}{\delta}\right)\right|=\frac{\left(\lambda_{1}-2 \lambda_{N}\right)^{2}}{\left(\lambda_{1}+2 \lambda_{N}\right)^{2}-8 \lambda_{N}^{2}} \approx \frac{\left(8-k^{2} h^{2}\right)^{2}}{-64+16 k^{2} h^{2}+k^{4} h^{4}},
$$

where we have again used the estimates $\lambda_{1} \approx-k^{2}$ and $\lambda_{N} \approx 4 / h^{2}-k^{2}$. Hence for an error reduction in the high frequencies by a factor $\epsilon$, one has to perform

$$
\nu \geq \frac{\log \epsilon}{\log \left(\left(8-k^{2} h^{2}\right)^{2} /\left(-64+16 k^{2} h^{2}+k^{4} h^{4}\right)\right)}
$$


smoothing steps. In the second case, $k h>\sqrt{6}$, the reduction factor on the high frequency modes is with the choice (2.20)

$$
\left|\left(1-\omega_{1} \frac{\lambda_{N}}{\delta}\right)\left(1-\omega_{2} \frac{\lambda_{N}}{\delta}\right)\right|=\frac{\left(\lambda_{1}-\lambda_{N}\right)^{2}}{\left(\lambda_{1}+7 \lambda_{N}\right)^{2}-32 \lambda_{N}^{2}} \approx \frac{1}{17-12 k^{2} h^{2}+2 k^{4} h^{4}} .
$$

For an error reduction in the high frequencies by a factor $\epsilon$, one has to perform in this last case

$$
\nu \geq \frac{-\log \epsilon}{\log \left(17-12 k^{2} h^{2}+2 k^{4} h^{4}\right)}
$$

smoothing steps.

We finally note that for $k h>\sqrt{6}$, one could also use the simple one-step Jacobi smoother, as it achieves a reduction factor of $1 / 3$ in that case. Using equioscillation to maximize damping on the high frequencies, the parameter should be chosen as

$$
\omega=\frac{4 \delta}{\lambda_{1}+3 \lambda_{N}}=\frac{2-k^{2} h^{2}}{3-k^{2} h^{2}-2 \sin ^{2} \frac{\pi h}{2}} .
$$

With this, one obtains a reduction in the high frequency components by at least a factor of

$$
\left|1-\omega \frac{\lambda_{N}}{\delta}\right|=\frac{\lambda_{1}-\lambda_{N}}{3 \lambda_{N}+\lambda_{1}} \approx \frac{1}{k^{2} h^{2}-3},
$$

using again the estimates $\lambda_{1} \approx-k^{2}$ and $\lambda_{N} \approx 4 / h^{2}-k^{2}$. Thus for an error reduction in the high-frequency components by a factor of $\epsilon$, one would have to perform

$$
\nu>\frac{-\log \epsilon}{\log \left(k^{2} h^{2}-3\right)}
$$

smoothing steps.

To summarize this section, we have shown by way of spectral analysis for our 1D model problem how damped Jacobi relaxation, one of the standard multigrid smoothers, breaks down at high wave numbers. As a possible remedy, we have shown how adapting the parameters of a two-step Jacobi iteration depending on the product $k h$ can yield a stable smoother which, however, on the resonance level, may require up to $O\left(k^{2}\right)$ smoothing steps to reduce the error on the oscillatory part of the spectrum by a fixed amount.

\section{Coarse Grid Correction}

The following spectral analysis of the coarse grid correction for the 1D case extends that in [33, Chapter 2] and [12, Chapter 5] for the Dirichlet-Laplacian model problem 
$(k=0)$. We consider problem (2.2) discretized using centered finite differences on a fine grid

$$
\Omega^{h}:=\left\{x_{j}=j h: j=0, \ldots, N+1\right\}
$$

with mesh width $h=\frac{1}{N+1}$ as well as a coarser grid $^{2}$

$$
\Omega^{H}:=\left\{x_{j}=j H: j=0, \ldots, n+1\right\}
$$

with twice the mesh width $H=2 h$ so that $N=2 n+1$. We introduce a grid transfer operator $I_{H}^{h}$ mapping functions $\boldsymbol{u}^{H}=\left[u_{0}^{H}, \ldots, u_{n+1}^{H}\right]$ defined on the coarse grid $\Omega^{H}$ to a function $I_{H}^{h} \boldsymbol{u}^{H}$ defined on the fine grid $\Omega^{h}$ using linear interpolation. This results in the linear mapping $\boldsymbol{u}^{H} \mapsto I_{H}^{h} \boldsymbol{u}^{H}$ defined by

$$
\left[I_{H}^{h} \boldsymbol{u}^{H}\right]_{j}=\left\{\begin{array}{ll}
{\left[\boldsymbol{u}^{H}\right]_{j / 2}} & \text { if } j \text { is even, } \\
\frac{1}{2}\left(\left[\boldsymbol{u}^{H}\right]_{(j-1) / 2}+\left[\boldsymbol{u}^{H}\right]_{(j+1) / 2}\right) & \text { if } j \text { is odd, }
\end{array} \quad j=0, \ldots, N+1,\right.
$$

and has the matrix representation

$$
\boldsymbol{I}_{H}^{h}=\frac{1}{2}\left[\begin{array}{lllll}
2 & & & & \\
1 & 1 & & & \\
& 2 & & & \\
& 1 & & & \\
& & \ddots & 1 & \\
& & & 2 & \\
& & & 1 & 1 \\
& & & 2
\end{array}\right] \in \mathbb{R}^{(N+2) \times(n+2)}
$$

with respect to the standard unit coordinate bases in $\mathbb{R}^{n+2}$ and $\mathbb{R}^{N+2}$.

Note that in case of Dirichlet boundary conditions at both interval ends, with which we begin our analysis, the values at the endpoints are omitted, resulting in vectors of length $N$ and $n$ on $\Omega^{h}$ and $\Omega^{H}$, respectively, as well as omitting of the first and last rows and columns in $\boldsymbol{I}_{H}^{h}$.

We analyze the mapping properties of the linear interpolation operator $I_{H}^{h}$ on the coarse-grid eigenvectors $\left\{\boldsymbol{v}_{j}^{H}\right\}_{j=1}^{n}$ of the discrete 1D Dirichlet-Laplacian, where

$$
\left[\boldsymbol{v}_{j}^{H}\right]_{\ell}=\sin (j \ell \pi H), \quad \ell=1, \ldots, n .
$$

\footnotetext{
${ }^{2}$ We remark that our choice of notation is intended to reflect that, on the fine grid, $N$ is large while $h$ is small and vice versa on the coarse grid.
} 


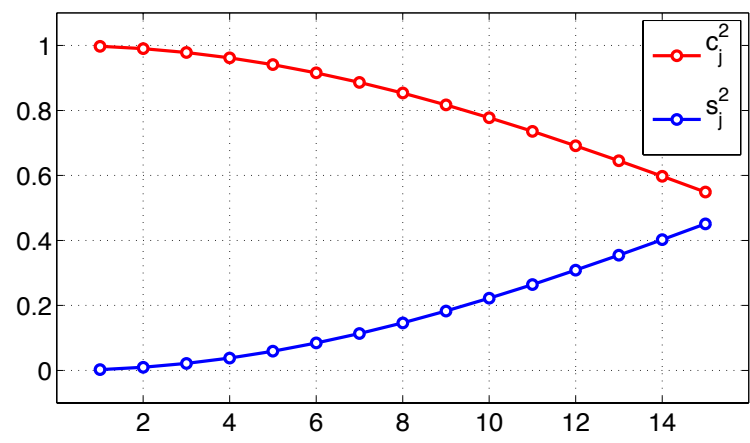

Figure 8. Coefficients $c_{j}^{2}$ and $s_{j}^{2}$ of the eigenvectors of the discrete 1D Dirichlet-Laplacian unter the linear interpolation operator for $N=31$, i.e., $n=15$.

Proposition 1. The coarse-grid eigenvectors are mapped by the interpolation operator $I_{H}^{h}$ according to

$$
I_{H}^{h} \boldsymbol{v}_{j}^{H}=c_{j}^{2} \boldsymbol{v}_{j}^{h}-s_{j}^{2} \boldsymbol{v}_{N+1-j}^{h}, \quad j=1, \ldots, n,
$$

where we define

$$
c_{j}:=\cos \frac{j \pi h}{2}, \quad s_{j}:=\sin \frac{j \pi h}{2}, \quad j=1, \ldots, n .
$$

In particular, $\boldsymbol{v}_{n+1}^{h}$ is not in the range of interpolation.

Proof. For any $j \in\{1, \ldots, n\}$ we distinguish the cases of odd and even index $\ell \in$ $\{1, \ldots, N\}$ corresponding to the two cases in the definition (3.1). In the first case we obtain using elementary trigonometric identities

$$
\begin{aligned}
{\left[I_{H}^{h} \boldsymbol{v}_{j}^{H}\right]_{\ell} } & \left.=\frac{1}{2}\left(\left[\boldsymbol{v}_{j}^{H}\right]_{(\ell-1) / 2}+\left[\boldsymbol{v}_{j}^{H}\right]_{(\ell+1) / 2}\right)=\frac{1}{2}\left(\sin \frac{j(\ell-1) \pi H}{2}\right)+\sin \frac{j(\ell+1) \pi H}{2}\right) \\
& =\frac{1}{2}(\sin (j(\ell-1) \pi h)+\sin (j(\ell+1) \pi h))=\cos (j \pi h) \sin (j \ell \pi h) \\
& =\left(c_{j}^{2}-s_{j}^{2}\right) \sin (j \ell \pi h)=c_{j}^{2} \sin (j \ell \pi h)-s_{j}^{2} \sin ((N+1-j) \ell \pi h) \\
& =c_{j}^{2}\left[\boldsymbol{v}_{j}^{h}\right]_{\ell}-s_{j}^{2}\left[\boldsymbol{v}_{N+1-j}^{h}\right]_{\ell} .
\end{aligned}
$$

For even $\ell$ we obtain

$$
\begin{aligned}
{\left[I_{H}^{h} \boldsymbol{v}_{j}^{H}\right]_{\ell} } & =\left[\boldsymbol{v}_{j}^{H}\right]_{\ell / 2}=\sin \frac{j \ell \pi H}{2}=\sin (j \ell \pi h)=\left(c_{j}^{2}+s_{j}^{2}\right) \sin (j \ell \pi h) \\
& =c_{j}^{2} \sin (j \ell \pi h)-s_{j}^{2} \sin ((N+1-j) \ell \pi h)=c_{j}^{2}\left[\boldsymbol{v}_{j}^{h}\right]_{\ell}-s_{j}^{2}\left[\boldsymbol{v}_{N+1-j}^{h}\right]_{\ell} .
\end{aligned}
$$


The coarse-grid modes $\boldsymbol{v}_{j}^{H}$ are thus mapped under the interpolation operator to a linear combination of their fine-grid counterparts $\boldsymbol{v}_{j}^{h}$ and a complementary mode $\boldsymbol{v}_{j^{\prime}}^{h}$ with index $j^{\prime}:=N+1-j$. Note the relations

$$
c_{j^{\prime}}=s_{j} \quad s_{j^{\prime}}=c_{j}, \quad j=1, \ldots, n,
$$

between complementary $s_{j}$ and $c_{j}$. Interpolating coarse-grid functions therefore always activates high-frequency modes on the fine grid, with a factor that is less than one but grows with $j$ (cf. Figure 8). This can be viewed as an aliasing phenomenon.

To transfer fine-grid functions to the coarse grid we employ the full weighting restriction operator

$$
I_{h}^{H}: \boldsymbol{u}^{h} \mapsto I_{h}^{H} \boldsymbol{u}^{h}
$$

defined by

$$
\left[I_{h}^{H} \boldsymbol{u}^{h}\right]_{j}=\frac{1}{4}\left(\left[\boldsymbol{u}^{h}\right]_{2 j-1}+2\left[\boldsymbol{u}^{h}\right]_{2 j}+\left[\boldsymbol{u}^{h}\right]_{2 j+1}\right), \quad j=1, \ldots, n
$$

The associated matrix representation is given by $\boldsymbol{I}_{h}^{H}=\frac{1}{2}\left[\boldsymbol{I}_{H}^{h}\right]^{\top}$. The restriction operator is thus seen to be the adjoint to the interpolation operator if one introduces on $\mathbb{R}^{n}$ and $\mathbb{R}^{N}$ the Euclidean inner product weighted by the mesh size $H$ and $h$, respectively. Denoting by $\mathscr{N}(\cdot)$ and $\mathscr{R}(\cdot)$ the null-space and range of a matrix, the basic relation

$$
\mathbb{R}^{N}=\mathscr{R}\left(\boldsymbol{I}_{H}^{h}\right) \oplus \mathscr{N}\left(\left[\boldsymbol{I}_{H}^{h}\right]^{\top}\right)=\mathscr{R}\left(\boldsymbol{I}_{H}^{h}\right) \oplus \mathscr{N}\left(\boldsymbol{I}_{h}^{H}\right)
$$

reveals that the range of interpolation and the null-space of the restriction are complementary linear subspaces of $\mathbb{R}^{N}$, which are also orthogonal with respect to the Euclidean inner product. Since the columns of $\boldsymbol{I}_{H}^{h}$ are seen to be linearly independent, the interpolation operator has full rank, which together with (3.5) implies

$$
\operatorname{dim} \mathscr{R}\left(\boldsymbol{I}_{H}^{h}\right)=n, \quad \operatorname{dim} \mathscr{N}\left(\boldsymbol{I}_{h}^{H}\right)=N-n=n+1 .
$$

Proposition 2. The fine-grid eigenvectors are mapped by the restriction operator $I_{h}^{H}$ according to

$$
\begin{aligned}
I_{h}^{H} \boldsymbol{v}_{j}^{h} & =c_{j}^{2} \boldsymbol{v}_{j}^{H}, & j=1, \ldots, n, \\
I_{h}^{H} \boldsymbol{v}_{N+1-j}^{h} & =-s_{j}^{2} \boldsymbol{v}_{j}^{H}, & j=1, \ldots, n, \\
I_{h}^{H} \boldsymbol{v}_{n+1}^{h} & =\boldsymbol{O} . &
\end{aligned}
$$


Proof. By (3.4) and elementary trigonometric relations we have for $j, \ell \in\{1, \ldots, n\}$

$$
\begin{aligned}
{\left[I_{h}^{H} \boldsymbol{v}_{j}^{h}\right]_{\ell} } & =\frac{1}{4}\left(\left[\boldsymbol{v}_{j}^{h}\right]_{2 \ell-1}+2\left[\boldsymbol{v}_{j}^{h}\right]_{2 \ell}+\left[\boldsymbol{v}_{j}^{h}\right]_{2 \ell+1}\right) \\
& =\frac{1}{4}(\sin ((2 \ell-1) j \pi h)+2 \sin (2 \ell j \pi h)+\sin ((2 \ell+1) j \pi h)) \\
& =\frac{1}{4}(2 \sin (2 \ell j \pi h)+2 \cos (j \pi h) \sin (2 \ell j \pi h)) \\
& =\frac{1}{2}(1+\cos (j \pi h)) \sin (2 \ell j \pi h) \\
& =\cos ^{2} \frac{j \pi h}{2} \sin (\ell j \pi H)=c_{j}^{2}\left[\boldsymbol{v}_{j}^{H}\right]_{\ell},
\end{aligned}
$$

which is (3.6a). For $j=n+1$ we have $2 j h=1$, implying $\sin (2 \ell j \pi h)=0 \forall \ell$, and thus the fourth equality above results in (3.6c). To show (3.6b) note first that

$$
\left[\boldsymbol{v}_{N+1-j}^{h}\right]_{\ell}=-(-1)^{\ell} \sin (j \ell \pi h), \quad j=1, \ldots, n ; \quad \ell=1, \ldots, N,
$$

and therefore

$$
\begin{aligned}
{\left[I_{h}^{H} \boldsymbol{v}_{N+1-j}^{h}\right]_{\ell} } & =\frac{1}{4}\left(2 \cos (j \pi h) \sin (j \ell \pi H)-(-1)^{2 \ell} 2 \sin (j \ell \pi H)\right) \\
& =\frac{1}{2}(\cos (j \pi h)-1) \sin (j \ell \pi H)=-\sin ^{2} \frac{j \pi h}{2} \sin (j \ell \pi H)=-s_{j}^{2}\left[\boldsymbol{v}_{j}^{H}\right]_{\ell} .
\end{aligned}
$$

In the standard "correction mode" of multigrid schemes for general linear problems the coarse-grid correction of an approximation $\boldsymbol{u}^{h}$ to the solution of (2.2) on the fine grid $\Omega^{h}$ is obtained by solving the error equation $\boldsymbol{A}_{h} \boldsymbol{e}^{h}=\boldsymbol{b}-\boldsymbol{A}_{h} \boldsymbol{u}^{h}=\boldsymbol{r}^{h}$ on the coarse grid. To this end, the residual is first restricted to the coarse grid, and a coarsegrid representation $\boldsymbol{A}_{H}$ of the differential operator used to obtain the approximation $\boldsymbol{A}_{H}^{-1} \boldsymbol{I}_{h}^{H} \boldsymbol{r}^{h}$ of the error $\boldsymbol{A}_{h}^{-1} \boldsymbol{r}^{h}$ on $\Omega^{H}$. The update is then obtained, after interpolating this error approximation to $\Omega^{h}$, as

$$
\boldsymbol{u}^{h} \leftarrow \boldsymbol{u}^{h}+\boldsymbol{I}_{H}^{h} \boldsymbol{A}_{H}^{-1} \boldsymbol{I}_{h}^{H}\left(\boldsymbol{b}-\boldsymbol{A}_{h} \boldsymbol{u}^{h}\right)
$$

with associated error propagation operator

$$
\boldsymbol{C}:=\boldsymbol{I}-\boldsymbol{I}_{H}^{h} \boldsymbol{A}_{H}^{-1} \boldsymbol{I}_{h}^{H} \boldsymbol{A}_{h} .
$$

When the so-called Galerkin condition is satisfied, i.e., when the coarse-grid operator is related to interpolation, restriction and the fine-grid operator by

$$
\boldsymbol{A}_{H}=\boldsymbol{I}_{h}^{H} \boldsymbol{A}_{h} \boldsymbol{I}_{H}^{h}
$$


then it is easily verified that $C^{2}=C$, i.e., $C$ is idempotent and thus a projection operator. More precisely, it is the complementary projection of $\boldsymbol{I}_{H}^{h} \boldsymbol{A}_{H}^{-1} \boldsymbol{I}_{h}^{H} \boldsymbol{A}_{h}$, the range of which is the range of interpolation $\mathscr{R}\left(\boldsymbol{I}_{H}^{h}\right)$. We conclude that the coarse-grid correction is without effect on error components outside the range of interpolation. In the same vein, coarse-grid correction is without effect for an error component whose associated residual vector, i.e., its image under $\boldsymbol{A}_{h}$, lies in the null-space of restriction. Moreover, the relation $\left(\boldsymbol{A}_{h} \boldsymbol{C}\right)^{\top}=\boldsymbol{A}_{h} \boldsymbol{C}$, which holds, e.g., when restriction is a scalar multiple of the transpose of interpolation ${ }^{3}$, implies that $\boldsymbol{C}$ is $\boldsymbol{A}_{h}$-selfadjoint, i.e., selfadjoint with respect to the inner product associated with $\boldsymbol{A}_{h}$ whenever $\boldsymbol{A}_{h}$ is symmetric and positive definite. In this case $\boldsymbol{C}$ is identified as the $\boldsymbol{A}_{h}$-orthogonal projection onto the $\boldsymbol{A}_{h}$-orthogonal complement of $\mathscr{R}\left(\boldsymbol{I}_{H}^{h}\right)$. We may thus write the direct sum (3.5) equivalently as the $\boldsymbol{A}_{h}$-orthogonal decomposition

$$
\mathbb{R}^{N}=\mathscr{R}\left(\boldsymbol{I}_{H}^{h}\right) \oplus \mathscr{N}\left(\boldsymbol{I}_{h}^{H} \boldsymbol{A}_{h}\right) .
$$

Returning to the 1D Helmholtz problem: in view of Propositions 1 and 2 the coarsegrid correction operator $C$ is seen to possess the invariant subspaces

$$
\operatorname{span}\left\{\boldsymbol{v}_{n+1}^{h}\right\} \quad \text { and } \quad \operatorname{span}\left\{\boldsymbol{v}_{j}^{h}, \boldsymbol{v}_{j^{\prime}}^{h}\right\}, \quad j^{\prime}=N+1-j, \quad j=1, \ldots, n .
$$

Noting that the eigenvalues of $\boldsymbol{A}_{h}$ and $\boldsymbol{A}_{H}$ are given by

$$
\lambda_{j}^{h}=\frac{4}{h^{2}} \sin ^{2} \frac{j \pi h}{2}-k^{2}, \quad j=1, \ldots, N,
$$

and

$$
\lambda_{j}^{H}=\frac{4}{H^{2}} \sin ^{2} \frac{j \pi H}{2}-k^{2}, \quad j=1, \ldots, n,
$$

respectively, the action of the coarse-grid correction operator on these invariant subspaces is given by

$$
\begin{aligned}
\boldsymbol{C}\left[\begin{array}{ll}
\boldsymbol{v}_{j}^{h} & \boldsymbol{v}_{j^{\prime}}^{h}
\end{array}\right] & =\left[\begin{array}{ll}
\boldsymbol{v}_{j}^{h} & \boldsymbol{v}_{j^{\prime}}^{h}
\end{array}\right] \boldsymbol{C}_{j}, \quad j=1, \ldots, n, \\
\boldsymbol{C} \boldsymbol{v}_{n+1}^{h} & =\boldsymbol{v}_{n+1}^{h},
\end{aligned}
$$

where

$$
\boldsymbol{C}_{j}=\left[\begin{array}{ll}
1 & 0 \\
0 & 1
\end{array}\right]-\left[\begin{array}{r}
c_{j}^{2} \\
-s_{j}^{2}
\end{array}\right] \frac{1}{\lambda_{j}^{H}}\left[\begin{array}{ll}
c_{j}^{2} & -s_{j}^{2}
\end{array}\right]\left[\begin{array}{cc}
\lambda_{j}^{h} & 0 \\
0 & \lambda_{j^{\prime}}^{h}
\end{array}\right]=\left[\begin{array}{cc}
1-c_{j}^{4} \frac{\lambda_{j}^{h}}{\lambda_{j}^{H}} & c_{j}^{2} s_{j}^{2} \frac{\lambda_{j^{\prime}}^{h}}{\lambda_{j}^{H}} \\
c_{j}^{2} s_{j}^{2} \frac{\lambda_{j}^{h}}{\lambda_{j}^{H}} & 1-s_{j}^{4} \frac{\lambda_{j^{\prime}}^{h}}{\lambda_{j}^{H}}
\end{array}\right] .
$$

\footnotetext{
${ }^{3}$ More generally, it is true if transpose is replaced by adjoint and restriction is adjoint to interpolation, where both adjoints are taken with respect to the same inner product.
} 
Proposition 3. The eigenvalues of the $2 \times 2$ blocks (3.10) representing the coarse-grid correction operator are given by

$$
\Lambda\left(\boldsymbol{C}_{j}\right)=\left\{1-\frac{c_{j}^{4} \lambda_{j}^{h}+s_{j}^{4} \lambda_{j^{\prime}}^{h}}{\lambda_{j}^{H}}, 1\right\}, \quad j=1, \ldots, n .
$$

A set of corresponding eigenvectors is given by

$$
\boldsymbol{w}_{j}^{(1)}=\left[\begin{array}{c}
c_{j}^{2} \\
-s_{j}^{2}
\end{array}\right] \quad \text { and } \quad \boldsymbol{w}_{j}^{(2)}=\left[\begin{array}{c}
s_{j}^{2} \lambda_{j^{\prime}}^{h} \\
c_{j}^{2} \lambda_{j}^{h}
\end{array}\right]=\frac{4}{h^{2}}\left[\begin{array}{c}
s_{j}^{2}\left(c_{j}^{2}-\left(\frac{h k}{2}\right)^{2}\right) \\
c_{j}^{2}\left(s_{j}^{2}-\left(\frac{h k}{2}\right)^{2}\right)
\end{array}\right],
$$

respectively.

Proof. In view of (3.9a), (3.9b) and (3.3), the quantities appearing in (3.10) may be written as

$$
\lambda_{j}^{h}=\frac{4}{h^{2}} s_{j}^{2}-k^{2}, \quad \lambda_{j^{\prime}}^{h}=\frac{4}{h^{2}} c_{j}^{2}-k^{2}, \quad \lambda_{j}^{H}=\frac{4}{h^{2}} s_{j}^{2} c_{j}^{2}-k^{2}, \quad j=1, \ldots, n .
$$

The characteristic polynomial of $\boldsymbol{C}_{j}$ is then

$$
\begin{aligned}
\operatorname{det}\left(\lambda \boldsymbol{I}-\boldsymbol{C}_{j}\right) & =\left(\lambda-1+c_{j}^{4} \frac{\lambda_{j}^{h}}{\lambda_{j}^{H}}\right)\left(\lambda-1+s_{j}^{4} \frac{\lambda_{j^{\prime}}^{h}}{\lambda_{j}^{H}}\right)-c_{j}^{4} s_{j}^{4} \frac{\lambda_{j^{\prime}}^{h} \lambda_{j}^{h}}{\left(\lambda_{j}^{H}\right)^{2}} \\
& =(\lambda-1)^{2}+(\lambda-1) \frac{c_{j}^{4} \lambda_{j}^{h}+s_{j}^{4} \lambda_{j^{\prime}}^{h}}{\lambda_{j}^{H}}=(\lambda-1)\left(\lambda-1+\frac{c_{j}^{4} \lambda_{j}^{h}+s_{j}^{4} \lambda_{j^{\prime}}^{h}}{\lambda_{j}^{H}}\right)
\end{aligned}
$$

revealing the eigenvalues. The eigenvectors are easily verified by direct calculation.

Note that the second eigenvalue and the first eigenvector of each $2 \times 2$ block $C_{j}$ are independent of $k$.

\subsection{The Laplacian}

For $k=0$ we observe

$$
\frac{\lambda_{j}^{h}}{\lambda_{j}^{H}}=\frac{4 s_{j}^{2}}{\left(2 s_{j} c_{j}\right)^{2}}=\frac{1}{c_{j}^{2}} \quad \text { as well as } \quad \frac{\lambda_{j^{\prime}}^{h}}{\lambda_{j}^{H}}=\frac{4 c_{j}^{2}}{\left(2 s_{j} c_{j}\right)^{2}}=\frac{1}{s_{j}^{2}}, \quad j=1, \ldots, n,
$$

and therefore directly from (3.10) we obtain

$$
\boldsymbol{C}_{j}=\left[\begin{array}{cc}
1-c_{j}^{2} & c_{j}^{2} \\
s_{j}^{2} & 1-s_{j}^{2}
\end{array}\right]=\left[\begin{array}{cc}
s_{j}^{2} & c_{j}^{2} \\
s_{j}^{2} & c_{j}^{2}
\end{array}\right], \quad j=1, \ldots, n .
$$


A matrix of the form $\boldsymbol{X}=\left[\begin{array}{ll}\xi & \eta \\ \xi & \eta\end{array}\right]$ has eigenvalues and spectral norm given by

$$
\begin{aligned}
\Lambda(\boldsymbol{X}) & =\{0, \xi+\eta\}, \\
\|\boldsymbol{X}\| & =\left\|\boldsymbol{X} \boldsymbol{X}^{\top}\right\|^{1 / 2}=\sqrt{\xi^{2}+\eta^{2}}\left\|\left[\begin{array}{ll}
1 & 1 \\
1 & 1
\end{array}\right]\right\|^{1 / 2}=\sqrt{\xi^{2}+\eta^{2}} \cdot \sqrt{2} .
\end{aligned}
$$

For $C_{j}$ we thus obtain

$$
\Lambda\left(\boldsymbol{C}_{j}\right)=\left\{0, s_{j}^{2}+c_{j}^{2}\right\}=\{0,1\}, \quad\left\|\boldsymbol{C}_{j}\right\|=\sqrt{2\left(s_{j}^{4}+c_{j}^{4}\right)}, \quad j=1, \ldots, n .
$$

From $s_{j}^{2} \in\left[0, \frac{1}{2}\right]$ for $j=1, \ldots, n$ we obtain the bound

$$
\left\|\boldsymbol{C}_{j}\right\| \leq \max _{0 \leq t \leq \frac{1}{2}} \sqrt{2\left[t^{2}+(1-t)^{2}\right]}=\sqrt{2}, \quad j=1, \ldots, n .
$$

By consequence, we also have $\Lambda(C)=\{0,1\}$, which reflects the fact that $C$ is a projection in case $k=0$. This is true since the Galerkin condition (3.8) holds if $\boldsymbol{A}_{h}$ and $\boldsymbol{A}_{H}$ result from the centered finite difference discretizations of the 1D Laplacian on the uniform meshes of widths $h$ and $H=2 h$. Note that the Galerkin property is lost for this discretization once $k>0$.

What we observe in the Laplacian case is the usual division of labor of classical multigrid methods. The coarse-grid correction has the task of eliminating the lowfrequency components of the error. In view of (3.12) for $k=0$, a set of eigenvectors of $\boldsymbol{C}_{j}$ associated with eigenvalues zero and one, i.e., with the null-space and range of the coarse-grid correction, is

$$
\boldsymbol{w}_{j}^{(1)}=\left[\begin{array}{c}
c_{j}^{2} \\
-s_{j}^{2}
\end{array}\right] \quad \text { and } \quad \boldsymbol{w}_{j}^{(2)}=\left[\begin{array}{l}
1 \\
1
\end{array}\right]
$$

Note that these are $\boldsymbol{A}_{h}$-orthogonal. For small values of the index $j$ the complementary eigenmodes $\boldsymbol{v}_{j}^{h}$ and $\boldsymbol{v}_{j^{\prime}}^{h}$ of $\boldsymbol{A}_{h}$ are very smooth and highly oscillatory, respectively. Moreover, since in this case $c_{j}^{2} \approx 1, s_{j}^{2} \approx 0$, the eigenmode $w_{j}^{(1)}$ eliminated by the coarse-grid correction is closely aligned with the low-frequency mode $\boldsymbol{v}_{j}^{h}$. This alignment becomes less as $j$ increases.

\subsection{The Helmholtz Operator}

In the Helmholtz case $k>0$ the eigenvalues of the coarse-grid correction operator are given by (3.11). Elementary manipulations reveal that the non-unit eigenvalues of $C$ are

$$
\gamma_{j}=\gamma_{j}(k h)=\frac{\frac{(k h)^{2}}{2}}{\left(\frac{k h}{2 s_{j} c_{j}}\right)^{2}-1}, \quad j=1, \ldots, n .
$$



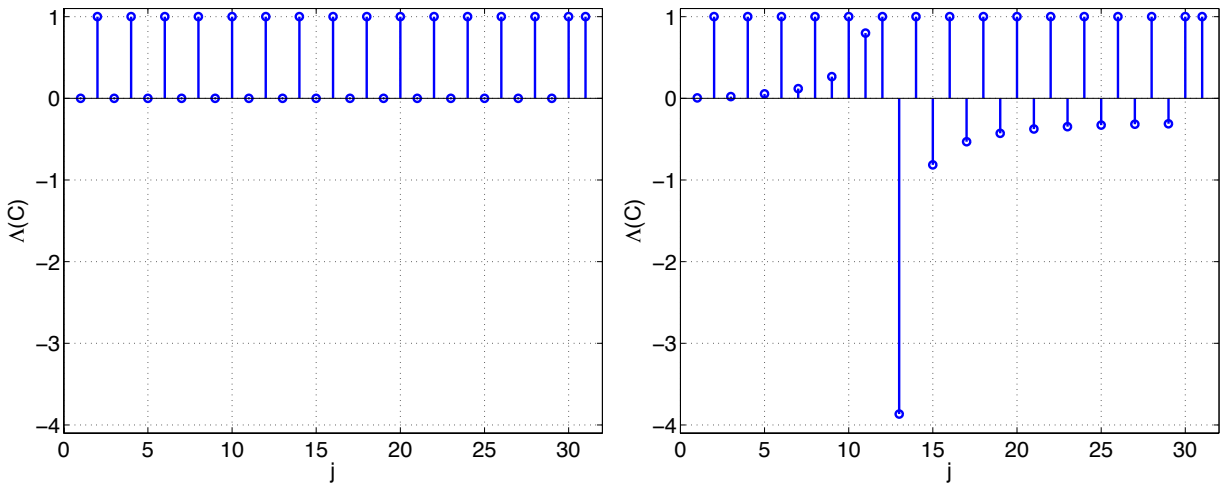

Figure 9. Eigenvalues of the coarse-grid operator with respect to a fine mesh with $h=$ $1 / 32$ for $k=0$ (left) and $k=6.3 \pi$ (right).
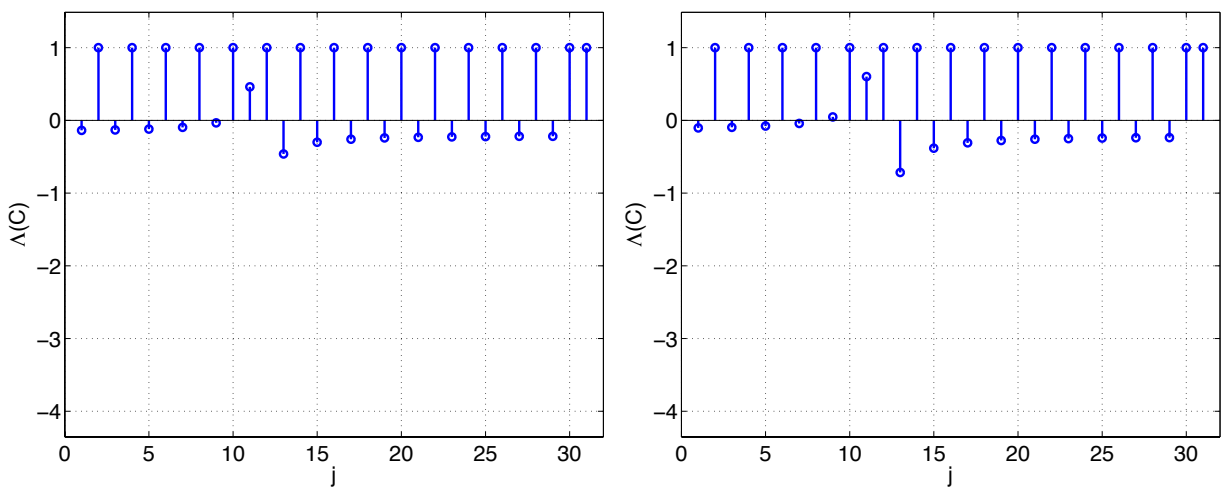

Figure 10. Eigenvalues of the coarse-grid operator with respect to a fine mesh with $h=1 / 32$ for $k=6.3 \pi$ using the modified wave number $\tilde{k}$ given in (3.15) (left) and (3.16) (right) in the coarse-grid Helmholtz operator.

For a fixed value of $k h>0$ this expression is seen to be a rational function of $s_{j} c_{j}$ with a simple pole whenever $2 s_{j} c_{j}=k h$. Since $2 s_{j} c_{j} \in(0,1)$ for $j \in\{1, \ldots, n\}$, this pole occurs near one of the indices $j$ whenever $k h \leq 1$.

This is illustrated in Figure 9 for a fine mesh containing $N=31$ interior points. The left plot shows a stem plot of the eigenvalues of the $2 \times 2$ blocks $C_{j}$ for the Laplacian, which consists of ones and zeros. On the right of Figure 9 we see the analogous plot for $k=6.3 \pi$. Note that the unit eigenvalues remain, but that the second eigenvalue of each pair is no longer zero. In particular, mode number 13 is amplified by a factor of nearly -4 . 


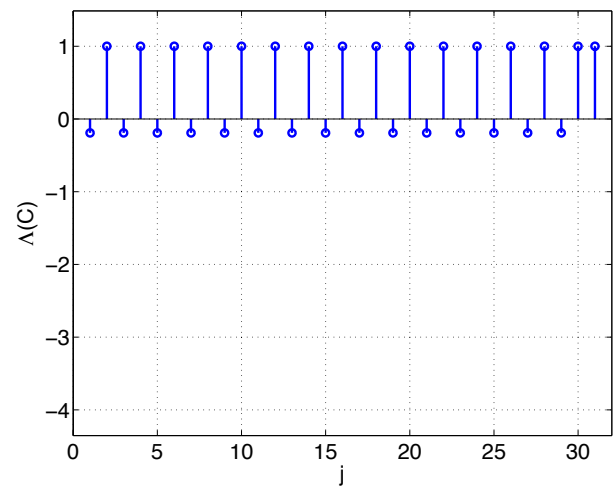

Figure 11. Eigenvalues of the coarse-grid operator with respect to a fine mesh with $h=1 / 32$ for $k=6.3 \pi$ using the modified wave number $\tilde{k}$ given in (3.17) in the coarsegrid Helmholtz operator.

\section{Dispersion Correction}

A simple device for obtaining a more effective coarse-grid correction for Helmholtz operators results from taking into account the dispersion properties of the discretization scheme. Dispersion analysis (cf. $[13,44])$ for discretization schemes is concerned with which propagating or standing waves solve a discrete homogeneous differential equation on a uniform grid extending over full space compared to the underlying continuous problem. For the centered finite-difference discretization of the 1D Helmholtz operator with constant $k$

$$
\mathscr{L} u \approx \frac{-u_{j-1}+2 u_{j}-u_{j+1}}{h^{2}}-k^{2} u_{j}
$$

plane-wave solutions $e^{i k^{h} x_{j}}$ of the discrete homogeneous Helmholtz equation possess a discrete wave number $k^{h}$ characterized by

$$
\frac{k^{h}}{k}=\frac{1}{k h} \arccos \left(1-\frac{k^{2} h^{2}}{2}\right)>1 .
$$

As a result, the discrete solution exhibits a phase lead with respect to the true solution, and this phase lead grows with $h$. In the same way, coarse-grid approximations in a multigrid hierarchy will be out of phase with fine-grid approximations. This suggests 'slowing down' the waves on coarse grids in order that the coarse-grid correction again be in phase with the fine-grid approximation. For our example, this is achieved by using a modified wave number $\tilde{k}$ in the coarse-grid Helmholtz operator defined by the requirement

$$
k^{H}=\frac{1}{H} \arccos \left(1-\frac{\tilde{k}^{2} H^{2}}{2}\right)=k, \quad \text { i.e. }, \quad \tilde{k}=\sqrt{\frac{2(1-\cos (k H))}{H^{2}}} .
$$


Alternatively, one could adjust the coarse-grid correction by matching the coarse-grid discrete wave number $k^{H}$ with the fine-grid discrete wave number $k^{h}$, which is obtained by choosing the modified wave number $\tilde{k}$ on the coarse grid to satisfy

$$
\tilde{k}^{H}=k^{h}
$$

i.e.,

$$
\frac{1}{H} \arccos \left(1-\frac{\tilde{k}^{2} H^{2}}{2}\right)=\frac{1}{h} \arccos \left(1-\frac{k^{2} h^{2}}{2}\right),
$$

or

$$
1-\frac{\tilde{k}^{2} H^{2}}{2}=\cos \left(\frac{H}{h} \arccos \left(1-\frac{k^{2} h^{2}}{2}\right)\right) .
$$

Noting $\frac{H}{h}=2$ and using the relation $\cos (2 \alpha)=2 \cos ^{2}(\alpha)-1$ this becomes

$$
1-\frac{\tilde{k}^{2} H^{2}}{2}=2\left(1-\frac{k^{2} h^{2}}{2}\right)^{2}-1,
$$

implying

$$
\tilde{k}=k \sqrt{1-\frac{k^{2} h^{2}}{4}} .
$$

Choosing a modified wave number according to (3.16) is also equivalent to avoiding a possible 'singularity' in the term $\lambda_{j}^{h} / \lambda_{j}^{H}$ in (3.10) by forcing the vanishing of $\lambda_{j}^{H}$ as a continuous function of $j$ to occur in the same location as for $\lambda_{j}^{h}$.

Figure 10 shows the eigenvalues of the coarse-grid correction operator depicted on the right of Figure 9 with the modified wave number (3.15) (left) and (3.16) (right) used on the coarse grid. The strong amplification of mode number 13 is seen to be much less severe, all non-unit eigenvalues now being less than one in modulus.

A closer analysis of the spectrum of the coarse-grid correction operator (3.10) reveals how the wave number $\tilde{k}$ on the coarse grid can be chosen to stabilize the correction yet further. Denoting the non-unit eigenvalue of $\boldsymbol{C}_{j}$ in (3.10) again by $\gamma_{j}$, upon changing the coarse-grid wave number from $k$ to $\tilde{k}$ we obtain

$$
\begin{aligned}
1-\gamma_{j} & =\frac{c_{j}^{4} \lambda_{j}^{h}+s_{j}^{4} \lambda_{j^{\prime}}^{h}}{\lambda_{j}^{H}}=\frac{c_{j}^{4}\left[\frac{4}{h^{2}} s_{j}^{2}-k^{2}\right]+s_{j}^{2}\left[\frac{4}{h^{2}} c_{j}^{2}-k^{2}\right]}{\frac{4}{h^{2}} s_{j}^{2} c_{j}^{2}-\tilde{k}^{2}} \\
& =\frac{s_{j}^{2} c_{j}^{2}-\left(s_{j}^{4}+c_{j}^{4}\right)\left(\frac{k h}{2}\right)^{2}}{s_{j}^{2} c_{j}^{2}-\left(\frac{\tilde{k} h}{2}\right)^{2}} .
\end{aligned}
$$

Setting $\tau_{j}:=s_{j}^{2} c_{j}^{2}$ and noting that $s_{j}^{4}+c_{j}^{4}=1-2 \tau_{j}$, this becomes

$$
1-\gamma_{j}=\frac{\tau_{j}-\left(1-2 \tau_{j}\right)\left(\frac{k h}{2}\right)^{2}}{\tau_{j}-\left(\frac{\tilde{k} h}{2}\right)^{2}}=\left(1+\frac{k^{2} h^{2}}{2}\right) \frac{\tau_{j}-\frac{k^{2} h^{2} / 4}{1+k^{2} h^{2} / 2}}{\tau_{j}-\frac{\tilde{k}^{2} h^{2}}{4}} .
$$


We observe that the non-unit eigenvalue of $\boldsymbol{C}_{j}$ can be made constant for all $j$ by adjusting $\tilde{k}$ to cancel the numerator and denominator on the right, which occurs for

$$
\tilde{k}=\frac{k}{\sqrt{1+\frac{k^{2} h^{2}}{2}}} .
$$

In this case the non-unit eigenvalue is

$$
\gamma_{j}=-\frac{k^{2} h^{2}}{2}
$$

Such a dispersion analysis can be carried out for all standard discretization schemes, and it is found that higher order schemes have much lower phase error (cf., e.g., [1]), making them a favorable choice also from the point of view of multigrid solvers. In higher dimensions higher order methods also possess nearly isotropic dispersion relations, a necessary requirement for (scalar) dispersion correction.

\section{Eigenvalues and Singular Values}

We compare the eigenvalues and singular values of the coarse grid correction operator for the same problem as above with wave numbers $k=0$ and $k=6.3 \pi$ on a mesh with $h=1 / 32$. For the positive wave number we compare the unmodified case as well as modified wave numbers according to (3.16) and (3.17), respectively.

Figure 12 shows the eigenfunctions and eigenvalues sorted increasingly. As a debugging measure, we computed these both by applying MATLAB's eig and svd routines to the matrix representing the complete coarse grid correction operator as well as to each $2 \times 2$ block or, in case of the eigenvalues, using (3.11). We observe that modifying the wave number according to (3.17) almost perfectly restores the zero-one spectrum of the coarse grid correction for the 1D Laplacian. However, this occurs at the price of a large singular value, leading to a large norm for the operator.

Figure 13 displayes the same eigen- and singular values ordered according to the invariant subspaces. The large singular value is seen to lie in the subspace with index $j=6$.

\section{Two-Grid Iteration}

The two-grid iteration combines one or more applications of the smoothing iteration with a coarse-grid correction. If $\nu_{1}$ and $\nu_{2}$ denote the number of pre- and postsmoothing steps carried out before and after the coarse-grid correction, the error propagation operator of the resulting two-grid operator is obtained as

$$
T=S^{\nu_{2}} C S^{\nu_{1}}
$$

Choosing the damped Jacobi iteration with relaxation factor $\omega$ as the smoothing operator, the results on the spectral analysis of the damped Jacobi smoother and coarse-grid 

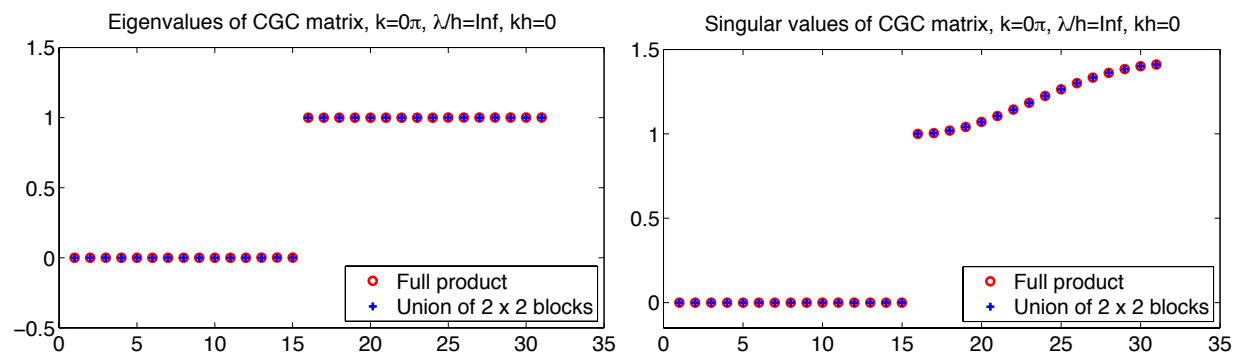

Eigenvalues of CGC matrix, $\mathrm{k}=6.3 \pi, \lambda / \mathrm{h}=10.2, \mathrm{kh}=0.62$
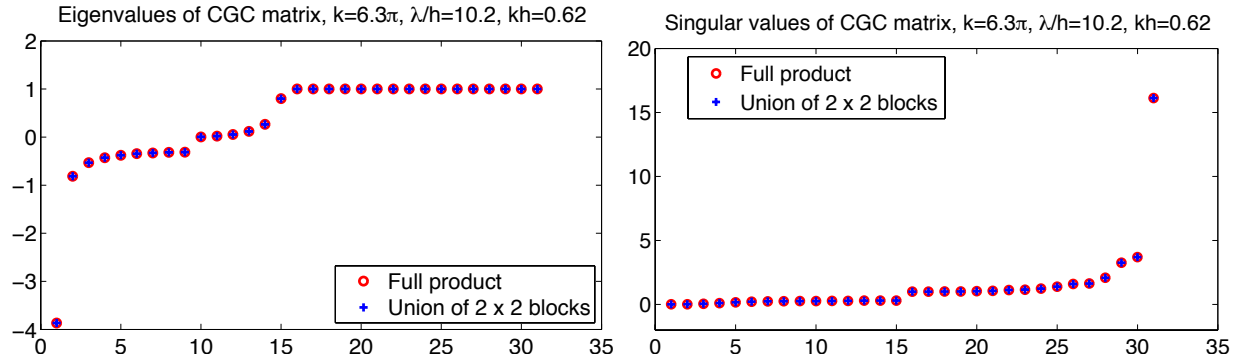

Singular values of CGC matrix, $k=6.3 \pi, \lambda / h=10.2, k h=0.62$
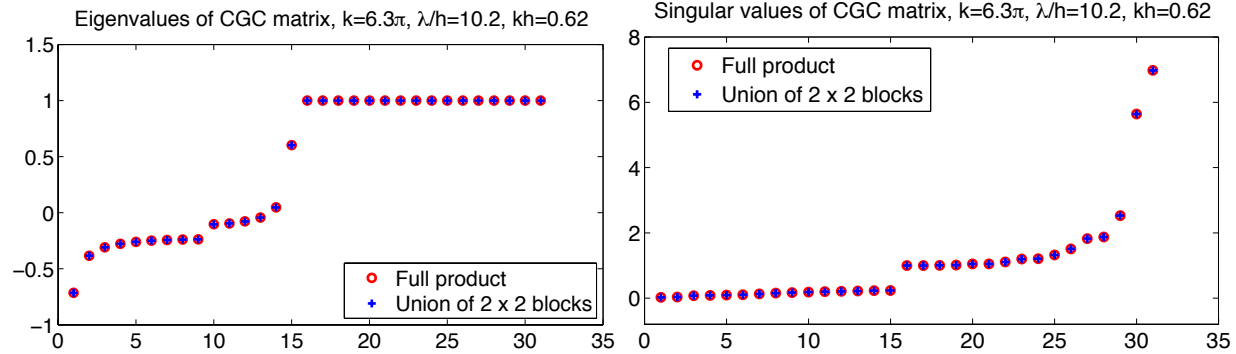

Eigenvalues of CGC matrix, $\mathrm{k}=6.3 \pi, \lambda / \mathrm{h}=10.2, \mathrm{kh}=0.62$

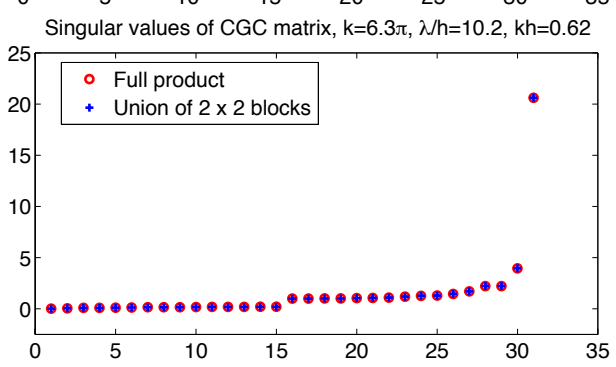

Figure 12. Eigenvalues (left) and singular values (right) of the coarse grid correction operator in the cases $k=0$ (row 1) and, for wave number $k=6.3 \pi$, the unmodified wave number (row 2), modified wave number according to (3.16) (row 3) and modified wave number according to (3.17) (row 4). 

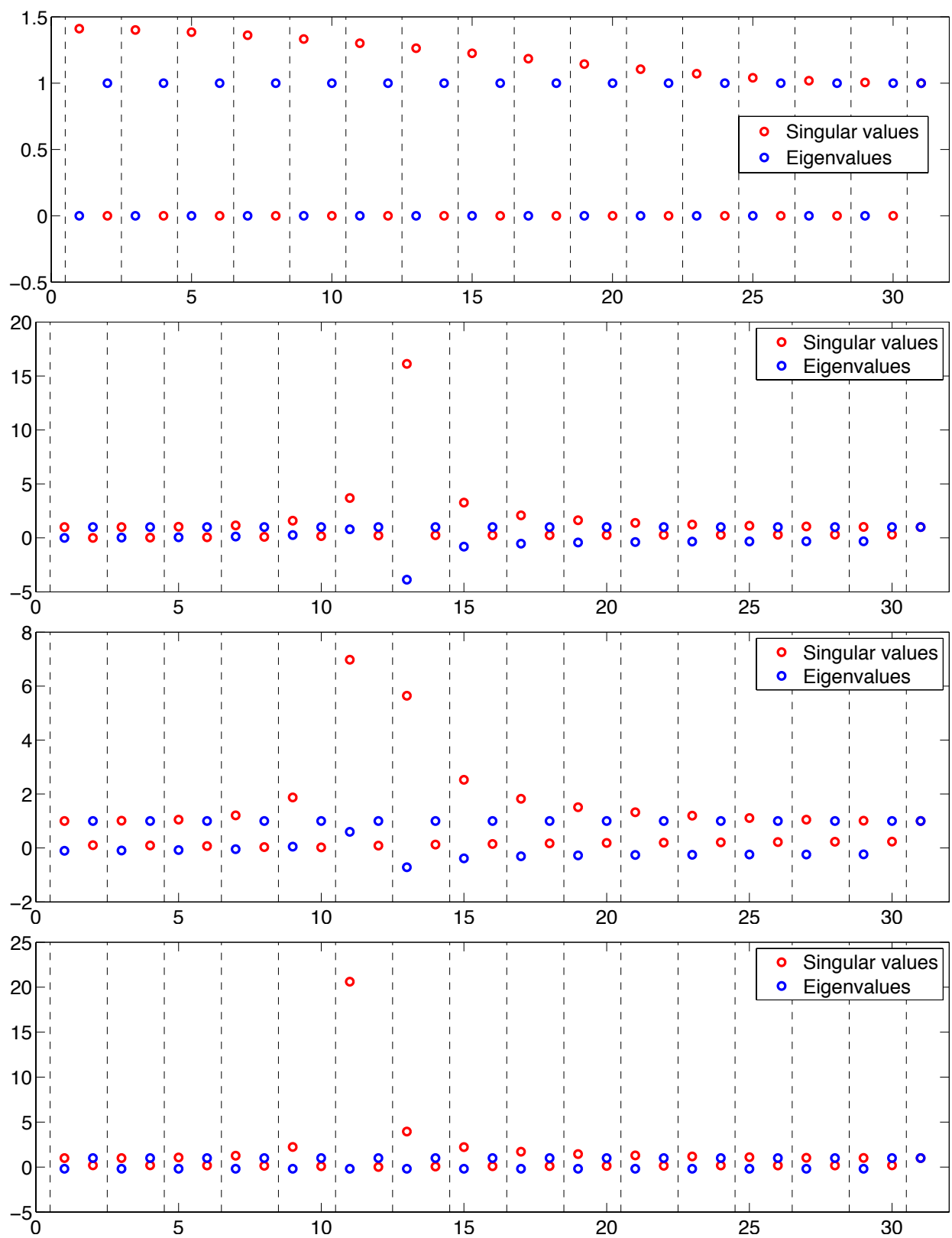

Figure 13. Eigenvalues and singular values of the coarse grid correction operator arranged by invariant two and one-dimensional subspaces in the cases $k=0$ (row 1) and, for wave number $k=6.3 \pi$, the unmodified wave number (row 2), modified wave number according to (3.16) (row 3) and modified wave number according to (3.17) (row 4). 
correction allow us to decompose the analysis of the two-grid operator into the subspaces

$$
\operatorname{span}\left\{\boldsymbol{v}_{1}, \boldsymbol{v}_{N}\right\}, \operatorname{span}\left\{\boldsymbol{v}_{2}, \boldsymbol{v}_{N-1}\right\}, \ldots, \operatorname{span}\left\{\boldsymbol{v}_{n}, \boldsymbol{v}_{n+2}\right\}, \operatorname{span}\left\{\boldsymbol{v}_{n+1}\right\}
$$

of $n$ pairs of complementary modes and the remaining 'middle mode' $\boldsymbol{v}_{n+1}$. The action of $T$ on these one- and two-dimensional subspaces is represented by the block diagonal matrix

$$
\boldsymbol{T}=\operatorname{diag}\left(\boldsymbol{T}_{1}, \ldots, \boldsymbol{T}_{n}, \boldsymbol{T}_{n+1}\right)
$$

with

$$
\boldsymbol{T}_{j}=\left[\begin{array}{cc}
\sigma_{j} & 0 \\
0 & \sigma_{j^{\prime}}
\end{array}\right]^{\nu_{2}}\left[\begin{array}{cc}
1-c_{j}^{4} \frac{\lambda_{j}^{h}}{\lambda_{j}^{H}} & c_{j}^{2} s_{j}^{2} \frac{\lambda_{j^{\prime}}^{h}}{\lambda_{j}^{H}} \\
c_{j}^{2} s_{j}^{2} \frac{\lambda_{j}^{h}}{\lambda_{j}^{H}} & 1-s_{j}^{4} \frac{\lambda_{j^{\prime}}^{h}}{\lambda_{j}^{H}}
\end{array}\right]\left[\begin{array}{cc}
\sigma_{j} & 0 \\
0 & \sigma_{j^{\prime}}
\end{array}\right]^{\nu_{1}} \quad j=1, \ldots, n,
$$

and

$$
\boldsymbol{T}_{n+1}=(1-\omega)^{\nu_{1}+\nu_{2}}
$$

the latter resulting from $\sigma_{n+1}=1-\omega$ (cf. (2.8)).

As a result, in the spectral basis $\left\{v_{j}^{h}\right\}_{j=1}^{N}$ of the discrete 1D Helmholtz equation the complete two-grid operator decouples into diagonal $2 \times 2$ blocks in addition to a trivial block for the middle mode $v_{n+1}^{h}$. We can therefore reduce the following analysis to the action of the two-grid iteration on the associated two-dimensional subspaces.

\subsection{The Laplacian}

We consider the case $k=0$, in which, due to (3.13), the $2 \times 2$ blocks in (4.1) simplify to

$$
\boldsymbol{T}_{j}=\left[\begin{array}{cc}
\sigma_{j} & 0 \\
0 & \sigma_{j^{\prime}}
\end{array}\right]^{\nu_{2}}\left[\begin{array}{cc}
s_{j}^{2} & c_{j}^{2} \\
s_{j}^{2} & c_{j}^{2}
\end{array}\right]\left[\begin{array}{cc}
\sigma_{j} & 0 \\
0 & \sigma_{j^{\prime}}
\end{array}\right]^{\nu_{1}} \quad \text { with } \quad \sigma_{j}=1-2 \omega s_{j}^{2}, \sigma_{j^{\prime}}=1-2 \omega c_{j}^{2} .
$$

A further simplification, considered in [33, Section $2.2 \mathrm{ff}$.], results from the (suboptimal) choice of $\omega=\frac{1}{2}$ for the smoothing parameter as well as $\nu_{1}=\nu, \nu_{2}=0$, in which case

$$
\boldsymbol{T}_{j}=\left[\begin{array}{cc}
s_{j}^{2} & c_{j}^{2} \\
s_{j}^{2} & c_{j}^{2}
\end{array}\right]\left[\begin{array}{cc}
c_{j}^{2} & 0 \\
0 & s_{j}^{2}
\end{array}\right]^{\nu}=\left[\begin{array}{cc}
s_{j}^{2} c_{j}^{2 \nu} & c_{j}^{2} s_{j}^{2 \nu} \\
s_{j}^{2} c_{j}^{2 \nu} & c_{j}^{2} s_{j}^{2 \nu}
\end{array}\right], \quad j=1, \ldots, n, \quad \boldsymbol{T}_{n+1}=\left(\frac{1}{2}\right)^{\nu} .
$$

Using (3.14a) we thus obtain for the spectral radius

$$
\rho\left(\boldsymbol{T}_{j}\right)=s_{j}^{2} c_{j}^{2 \nu}+c_{j}^{2} s_{j}^{2 \nu}, \quad j=1, \ldots, n, \quad \rho\left(\boldsymbol{T}_{n+1}\right)=2^{-\nu} .
$$




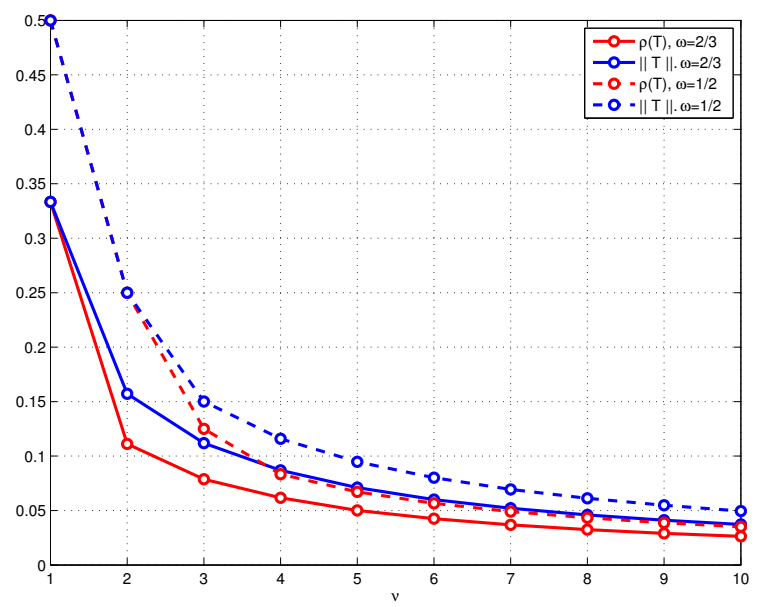

Figure 14. Spectral radius and spectral norm of the two-grid operator for the 1D model problem with $k=0$ using $\nu$ steps of pre-smoothing with optimal relaxation parameter $\omega=2 / 3$ (solid lines) and $\omega=1 / 2$ (dashed lines).

Noting that $c_{j}^{2}=1-s_{j}^{2}$ and $s_{j}^{2} \in\left[0, \frac{1}{2}\right]$ for all $j$, we obtain the upper bound

$$
\rho\left(\boldsymbol{T}_{j}\right) \leq R_{\nu}:=\max _{0 \leq t \leq \frac{1}{2}} R_{\nu}(t), \quad R_{\nu}(t):=t(1-t)^{\nu}+(1-t) t^{\nu}
$$

for $j=1, \ldots, n$. Since $R_{\nu}\left(\frac{1}{2}\right)=\left(\frac{1}{2}\right)^{\nu}$ this bound holds also for $\boldsymbol{T}_{n+1}$.

A common upper bound for the spectral norms $\left\|\boldsymbol{T}_{j}\right\|$ is obtained in an analogous way using (3.14b) as

$$
\left\|\boldsymbol{T}_{j}\right\| \leq N_{\nu}:=\max _{0 \leq t \leq \frac{1}{2}} N_{\nu}(t), \quad N_{\nu}(t):=\sqrt{2\left[t^{2}(1-t)^{2 \nu}+(1-t)^{2} t^{2 \nu}\right]}
$$

which, in view of $N_{\nu}\left(\frac{1}{2}\right)=\left(\frac{1}{2}\right)^{\nu}$, is again also valid for $\boldsymbol{T}_{n+1}$.

Choosing the optimal smoothing parameter $\omega=\frac{2}{3}$ as well as $\nu_{1}=\nu, \nu_{2}=0$ results in

$$
\boldsymbol{T}_{j}=\left[\begin{array}{cc}
s_{j}^{2} \sigma_{j}^{\nu} & c_{j}^{2} \sigma_{j^{\prime}}^{\nu} \\
s_{j}^{2} \sigma_{j}^{\nu} & c_{j}^{2} \sigma_{j^{\prime}}^{\nu}
\end{array}\right], \quad j=1, \ldots, n, \quad \boldsymbol{T}_{n+1}=\left(\frac{1}{3}\right)^{\nu}
$$

where now

$$
\sigma_{j}=\frac{1}{3}\left(3-4 s_{j}^{2}\right), \quad \sigma_{j^{\prime}}=\frac{1}{3}\left(4 s_{j}^{2}-1\right), \quad j=1, \ldots, n .
$$

Proceeding as above, this results in the upper bound

$$
\rho\left(\boldsymbol{T}_{j}\right) \leq \tilde{R}_{\nu}:=\max _{0 \leq t \leq \frac{1}{2}} \tilde{R}_{\nu}(t), \quad \tilde{R}_{\nu}(t):=t\left(\frac{3-4 t}{3}\right)^{\nu}+(1-t)\left(\frac{4 t-1}{3}\right)^{\nu}
$$




\begin{tabular}{c|c|c|c|c}
$\nu \backslash \rho(\boldsymbol{T})$ & $k=0$ & $k=1.3 \pi$ & $k=4.3 \pi$ & $k=6.3 \pi$ \\
\hline 1 & 0.3333 & 0.3364 & 0.4093 & 0.8857 \\
2 & 0.1111 & 0.1170 & 0.2391 & 1.8530 \\
3 & 0.0787 & 0.0779 & 0.2623 & 1.6455 \\
4 & 0.0617 & 0.0613 & 0.2481 & 1.6349 \\
5 & 0.0501 & 0.0493 & 0.2561 & 1.5832 \\
10 & 0.0263 & 0.0256 & 0.2668 & 1.3797 \\
\hline
\end{tabular}

Table 1. Spectral radius of the two-grid operator for the Helmholtz equation with $h=$ $1 / 32$ for varying wave number $k$ and (pre-) smoothing step number $\nu$.

for all $j=1, \ldots, n$ and, since $\tilde{R}_{\nu}\left(\frac{1}{2}\right)=\left(\frac{1}{3}\right)^{\nu}$, also for $\boldsymbol{T}_{n+1}$. For the spectral norm we obtain the bound

$$
\left\|\boldsymbol{T}_{j}\right\| \leq \tilde{N}_{\nu}:=\max _{0 \leq t \leq \frac{1}{2}} \tilde{N}_{\nu}(t), \quad \tilde{N}_{\nu}(t):=\sqrt{2\left[t^{2}\left(\frac{3-4 t}{3}\right)^{2 \nu}+(1-t)^{2}\left(\frac{4 t-1}{3}\right)^{2 \nu}\right]}
$$

which holds for all $j=1 \ldots, n+1$ due to $\tilde{N}_{\nu}\left(\frac{1}{2}\right)=\left(\frac{1}{3}\right)^{\nu}$.

We show in Figure 14 the spectral radii and 2-norms of the two-grid operator as a function of the number of smoothing steps for the case of Laplace's equation with both the optimal damping $\omega=2 / 3$ and the suboptimal damping $\omega=1 / 2$. Clearly, it is worthwhile to use the optimal damping parameter, especially when the number of smoothing steps is small.

\subsection{The Helmholtz Operator}

Table 1 gives the spectral radius of the two-grid operator for the Helmholtz equation with $\nu$ steps of pre-smoothing using damped Jacobi for a range of wave numbers $k$. We observe that the iteration is divergent for $k=6 \pi$, which corresponds to a resolution of roughly 10 points per wavelength. Moreover, while additional smoothing steps resulted in a faster convergence rate for $k$ close to zero, this is no longer the case for higher wave numbers.

In order to improve the situation, we note that we have two parameters, the damping parameter $\omega$ from the smoothing analysis and the shifted wave number $\tilde{k}$ we can choose in the coarse grid correction. We thus first investigate numerically whether a better choice is possible for these parameters. This choice needs to guarantee that each block $\boldsymbol{T}_{j}$ in (4.1) is a contraction, i.e. its eigenvalues must be smaller than one. If, in addition, the eigenvalues are distinct, then each block $\boldsymbol{T}_{j}$ is diagonalizable and the convergence rate determined by the eigenvalues is not only asymptotic, but visible right from the start, up to a constant given by the condition of the eigenvectors. After 


\begin{tabular}{c|c|c|c}
$\nu \backslash \rho(\boldsymbol{T})$ & $k=1.3 \pi$ & $k=4.3 \pi$ & $k=6.3 \pi$ \\
\hline 1 & $0.3302(4.4660,0.6632)$ & $0.3605(13.6088,0.6322)$ & $0.4046(19.1171,0.5875)$ \\
2 & $0.1129(4.1825,0.6637)$ & $0.1358(13.2518,0.6294)$ & $0.1602(18.7491,0.5959)$ \\
3 & $0.0708(4.1382,0.7040)$ & $0.0738(13.2373,0.6355)$ & $0.1184(18.9766,0.5076)$ \\
4 & $0.0590(4.1309,0.7030)$ & $0.0544(13.2857,0.6544)$ & $0.1207(18.9835,0.4098)$ \\
5 & $0.0394(4.1093,0.7583)$ & $0.0475(13.2537,0.6135)$ & $0.1239(18.9881,0.3552)$ \\
10 & $0.0226(4.0963,0.7797)$ & $0.0477(13.2570,0.3243)$ & $0.1342(18.9773,0.2036)$ \\
\hline
\end{tabular}

Table 2. Spectral radius of the two-grid operator for the Helmholtz equation with $h=1 / 32$ for varying wave number $k$ and (pre-) smoothing step number $\nu$, using optimized coarse wave number and relaxation parameter $(\tilde{k}, \omega)$ shown in parenthesis. For comparison, the original values are $k=1.3 \pi=4.0841$ with $\omega=\frac{2-h^{2} k^{2}}{3-h^{2} k^{2}}=0.6648$, $k=4.3 \pi=13.5088$ with $\omega=\frac{2-h^{2} k^{2}}{3-h^{2} k^{2}}=0.6456$, and $k=6.3 \pi=20.1062$ with $\omega=\frac{2-h^{2} k^{2}}{3-h^{2} k^{2}}=0.6179$.

some simplification, we find for the eigenvalues $\mu_{1,2}\left(\boldsymbol{T}_{j}\right)$ the formula

$$
\begin{aligned}
\mu_{1,2}\left(\boldsymbol{T}_{j}\right) & =\frac{\sigma_{j}^{\nu}\left(\lambda_{j}^{H}-c_{j}^{4} \lambda_{j}^{h}\right)+\sigma_{j^{\prime}}^{\nu}\left(\lambda_{j}^{H}-s_{j}^{4} \lambda_{j^{\prime}}^{h}\right) \pm \sqrt{D}}{2 \lambda_{j}^{H}}, \\
D & =\sigma_{j}^{2 \nu}\left(\lambda_{j}^{H}-c_{j}^{4} \lambda_{j}^{h}\right)^{2}+\sigma_{j^{\prime}}^{2 \nu}\left(\lambda_{j}^{H}-s_{j}^{4} \lambda_{j^{\prime}}^{h}\right)^{2} \\
& +2 \sigma_{j}^{\nu} \sigma_{j^{\prime}}^{\nu}\left(\lambda_{j}^{H} s_{j}^{4} \lambda_{j^{\prime}}^{h}+\lambda_{j}^{H} c_{j}^{4} \lambda_{j}^{h}+\lambda_{j^{\prime}}^{h} \lambda_{j}^{h} c_{j}^{4} s_{j}^{4}-\left(\lambda_{j}^{H}\right)^{2}\right),
\end{aligned}
$$

where we set $\nu:=\nu_{1}+\nu_{2}$, since only the sum appears in the eigenvalues. We now use the free parameters $\omega$ and $\tilde{k}$ to minimize numerically the maximum of $\left|\mu_{1,2}\right|$ over all $j=1,2, \ldots, n$ in order to test if this leads to a working algorithm. Doing so, we obtain the results shown in Table 2 . We see that it is indeed possible to obtain a converging multigrid algorithm in 1D using this approach. But what happens at higher wave numbers $k$ ?

In order to investigate this, we fix the mesh size to $h=1 / 32$, and apply our two-grid analysis for increasing wave number $k$, reflecting a typical realistic situation where, in a multigrid algorithm for a problem with large wave number $k$, one would reach such a situation after several coarsening steps. We must, however, be careful not to generate singular problems due to the Dirichlet boundary condition assumption in our analysis. To avoid this, we choose the particular wave number sequence

$$
k_{j}=\sqrt{\frac{2}{h^{2}}\left(\sin ^{2} \frac{(j-1) \pi h}{2}+\sin ^{2} \frac{j \pi h}{2}\right)}, \quad j=1,2, \ldots, N,
$$

placing $k_{j}^{2}$ exactly between two Dirichlet eigenvalues. This yields $N$ Helmholtz problems, and we apply to each of these the two-grid analysis. In Figure 15, we show as a 
function of $k_{j}$ what spectral radius one obtains with the various choices of components in the two-grid method.

The first curve corresponds to the classical two-grid method without modifying the wave number, and using ten smoothing steps (which would correspond to five pre- and five post-smoothing steps) of the one-step Jacobi smoother with the relaxation parameter appropriate for the Helmholtz equation given in (2.9). We see that this method diverges as soon as the wave number $k$ is larger than about 18, which corresponds to about $2 \pi /(k h) \approx 11$ points per wavelength, and is consistent with the observation in Table 1.

The second curve shows the spectral radius of the classical two-grid method with modified wave number according to the theoretical shift proposed in (3.16). We see that the two-grid algorithm is already performing somewhat better, a contraction now being obtained up to wave number $k=23$, corresponding to $2 \pi /(k h) \approx 9$ points per wavelength.

Numerically optimizing the shift by minimizing the spectral radius gives a slightly better contraction at this wave number, but does not lead to a convergent method at higher wave numbers, as confirmed by the third, red curve. One needs to modify the smoother.

The next three curves result when using the two-step Jacobi smoother with the number of smoothing steps given in (2.15) from Remark 2.2 and (2.18) from Remark 2.3, requiring a reduction by a factor of 10 in the high-frequency part of the spectrum. In the fourth curve we see that, without the shift, it does not help to use the convergent two step Jacobi smoother: as for the classical one-step Jacobi smoother, the two-grid method diverges as soon as one has fewer than about 11 points per wavelength resolution. This changes fundamentally, however, when one also uses the shifted wave number $k$ according to (3.16): now the two-grid algorithm is convergent for all wave numbers up to about $k=40$, which corresponds to about $2 \pi /(k h) \approx 5$ points per wavelength. Afterward, the shift becomes counter productive, and the shifted two grid algorithm diverges. However, in that regime, the unshifted algorithm does converge, and the numerical optimization of the shift indicates that one should indeed not shift as soon as the resolution has become less than about 5 points per wavelength. This does also make sense intuitively since, at that resolution, the corresponding coarse problem has now become (negative) definite, and is no longer a wave problem.

We show now in Figure 16 the various shifts that were used in these experiments. We see that the theoretical shifting formula (3.16) is very good for the two-grid method with the two-step Jacobi smoother, as long as the wave number is smaller than $k \approx 40$, which corresponds to 5 points per wave length. For higher values of $k$ the theoretical shift becomes meaningless, and the unshifted problem should be used.

We finally show in Figure 17 the number of smoothing steps that were used for the spectral radii shown in Figure 15. The graph corresponds to the theoretically required smoothing steps (2.15) and (2.18) to obtain a reduction by a factor of 10 in the highfrequency part of the spectrum. 


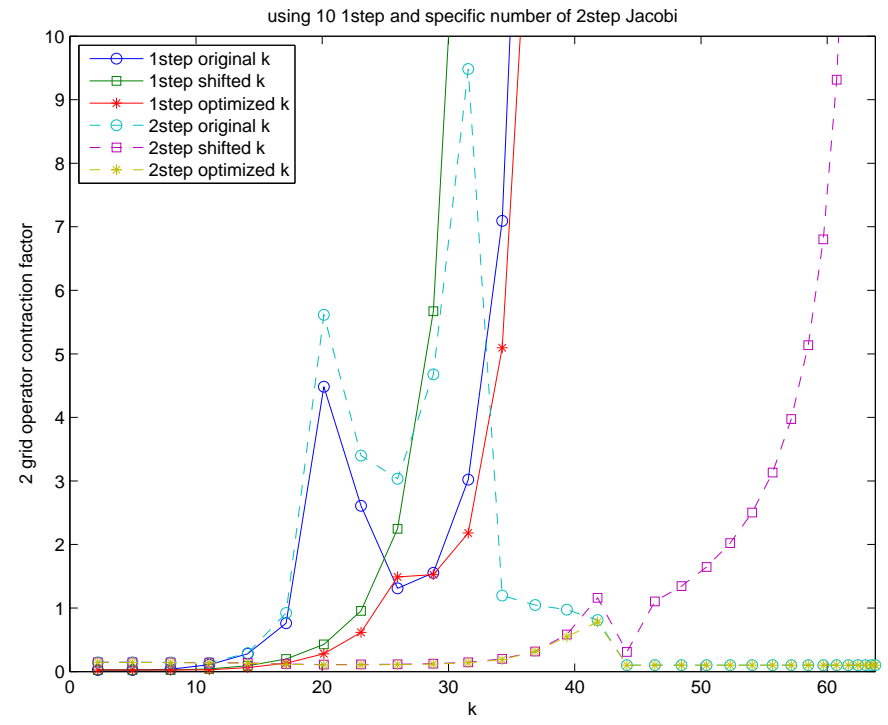

Figure 15. Spectral radii of the two grid algorithm using various components, for a fixed mesh size $h$, and increasing wave number $k$.

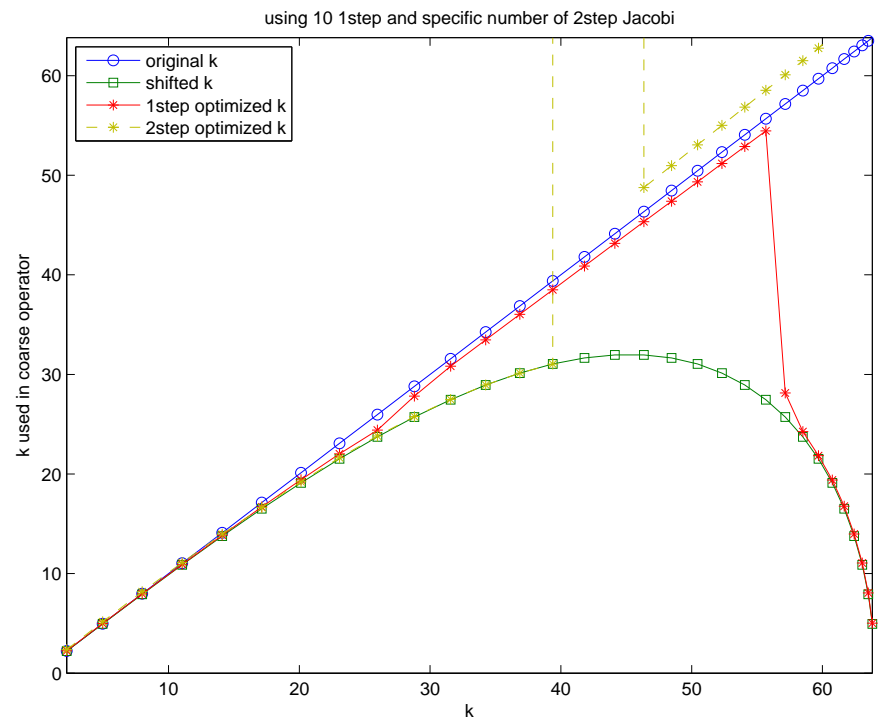

Figure 16. Shifts used in the two grid algorithm of Figure 15. 


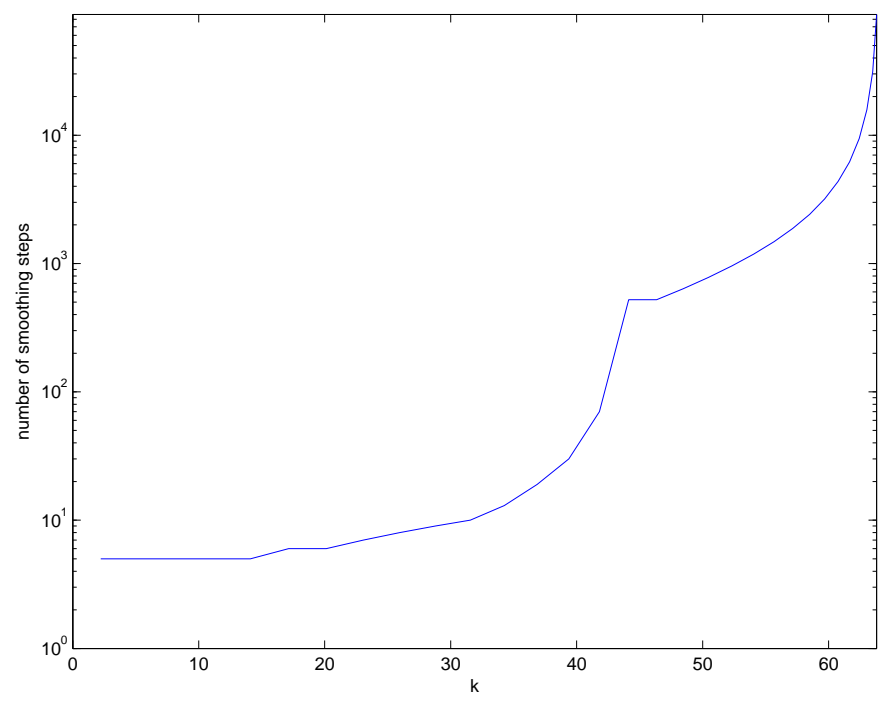

Figure 17. Number of smoothing steps used in the two-step Jacobi smoother for the two-grid algorithm of Figure 15.

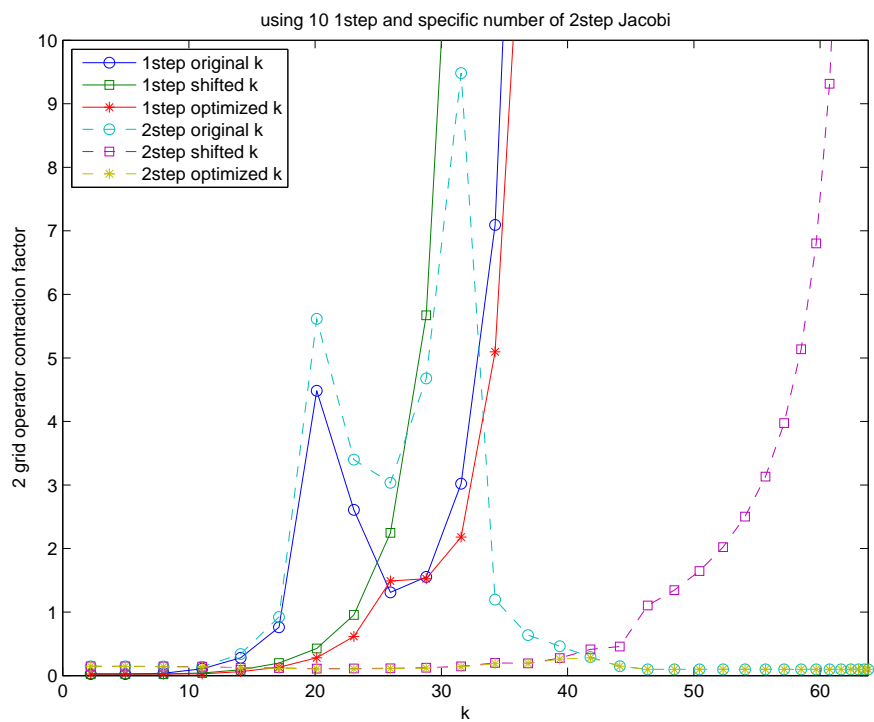

Figure 18. Contraction factors of the two grid algorithm using various components, for a fixed mesh size $h$, and increasing wave number $k$, now using the $O\left(k^{2}\right)$ smoothing steps already a bit before the resonance level. 


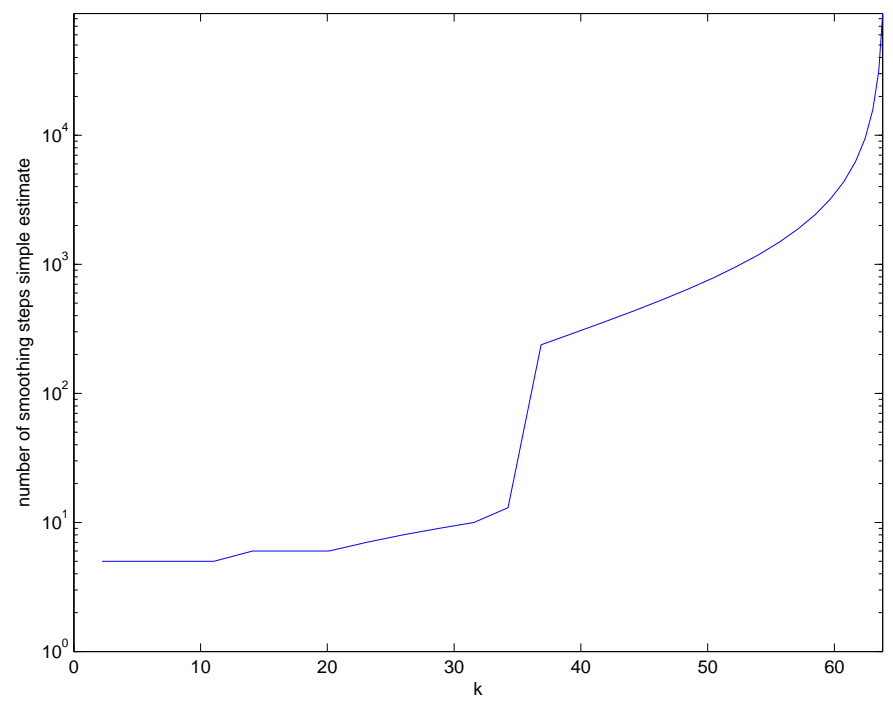

Figure 19. Number of smoothing steps used in the two step Jacobi smoother for the two grid algorithm of Figure 18.

We see that the number of smoothing steps is moderate for values of the wave number less that about 40, i.e., 5 points per wave length, but then increases dramatically to the order of about 1000, on the level where $k$ gets close to or into the resonance level. This is, however, not a surprise if one looks at the smoothing curve in Figure 6: clearly the smoother has difficulties to smooth close to and in the resonance level.

When taking a final look at Figure 15 again, we see that the shifted two-grid method with two-step Jacobi smoother seems to have difficulties to contract around $k=38-$ 44. This is, however, only due to our estimate in Remark 2.2 for the smoothing steps needed in the two-step Jacobi smoother, which was based on the smoother alone. For the case $k$ just above 40, this estimate leads to 71 smoothing steps, which is not quite enough, since the shift for the coarse problem starts to lose its effectiveness. We show in Figure 18 the same sequence of experiments again, but now using the estimate for the number of smoothing steps in the resonance level already for $k h>\sqrt{2}-0.3$, to overcome the difficulty of the coarse grid correction just before the shift becomes completely ineffective. The value 0.3 is just chosen from looking at these numerical experiments, an analysis would need to take into account the estimates for $\mu_{1,2}$ given in (4.2). Using more smoothing steps a bit before the resonance level leads to the results shown in Figure 18. The precise number of steps used is shown in Figure 19.

We see that it is indeed possible with this strategy to obtain a uniformly convergent two-grid algorithm for all possible values of $k$ with the mesh resolution $h=1 / 32$.

We show in Figures 20 and 21 the same type of experiment, but now for a finer mesh resolution $h=1 / 128$. In Figure 21, we see that in the range of $k$ between about 


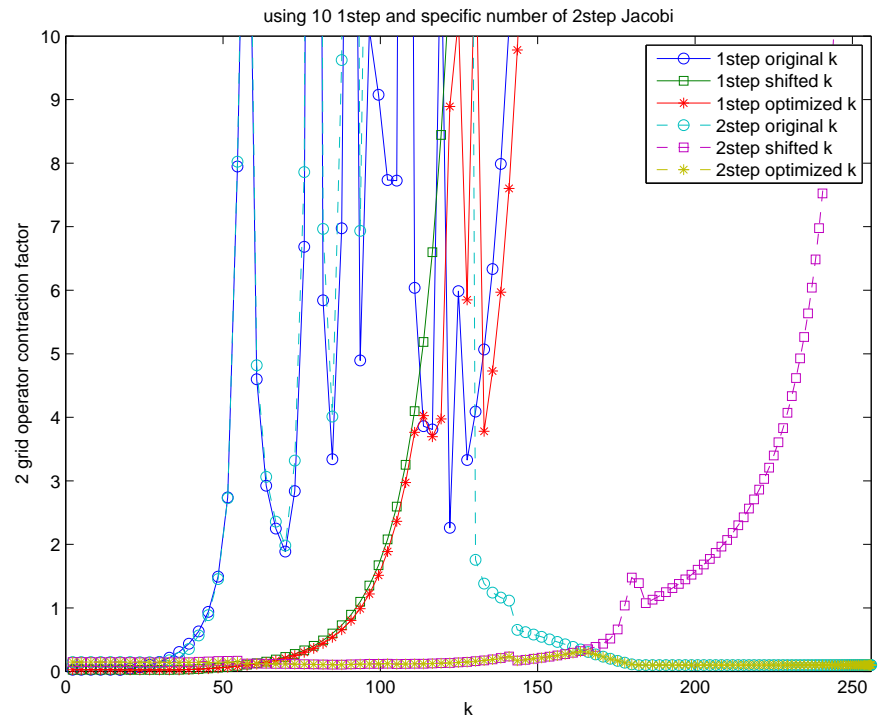

Figure 20. Contraction factors of the two grid algorithm using various components, for a fixed mesh size $h=1 / 128$, and increasing wave number $k$.

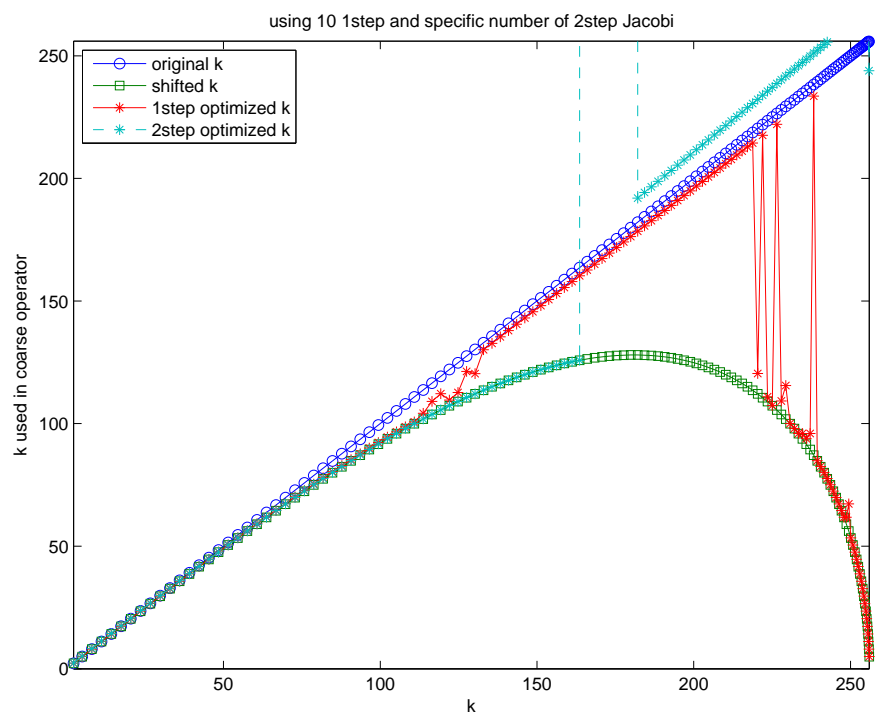

Figure 21. Shifts used in the two grid algorithm of Figure 20. 


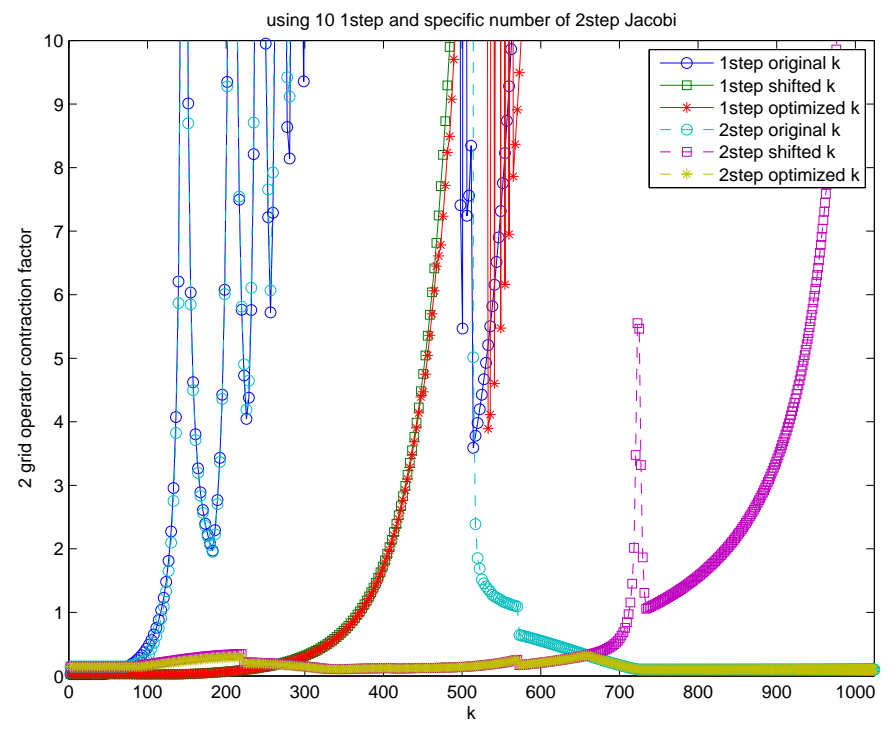

Figure 22. Contraction factors of the two grid algorithm using various components, for a fixed mesh size $h=1 / 512$, and increasing wave number $k$.

130 and 180, both the shifted and unshifted coarse problems function, there is a zone of overlap, which is very reassuring.

We finally show in Figure 22 the case of $h=1 / 512$. From these experiments, we can see that a reasonable strategy for a multigrid algorithm for the Helmholtz equation in 1D is to simply use a classical one-step Jacobi smoother and a shifted coarse problem, as long as the wave number and mesh size satisfy $h k<0.5$ for the current level mesh size $h$, which means we have about 13 points per wave length or more. For $0.5 \leq k h<1.25$, one has to switch to the two-step Jacobi smoother, and use a shifted coarse problem, and as soon as $k h \geq 1.25$, one should not use a shifted coarse problem any more, and needs to use $O\left(k^{2}\right)$ smoothing steps of the two-step Jacobi smoother, or a more effective smoothing method based on Chebyshev polynomials or a Krylov method.

\section{Numerical Examples}

In this section we describe some numerical experiments with two model problems. The first (cf. (2.2)) is the Helmholtz equation

$$
\begin{aligned}
& -u^{\prime \prime}-k^{2} u=f, \text { on }(0,1) \\
& u(0)=u(1)=0,
\end{aligned}
$$

with constant wave number $k>0$, homogeneous Dirichlet boundary conditions and a source term $f$. This may be viewed as a cavity problem in one dimension in view of 
the fact that the negative 1D Dirichlet-Laplacian has positive eigenvalues leading to a singular operator for the discrete values

$$
k=j \pi, \quad j \in \mathbb{N},
$$

the so-called resonance values of the wave number $k$. Problem (5.1) is well-posed for all $0<k \neq j \pi$, and discretizations thereof are well-behaved for sufficiently fine resolution.

As a second 1D model problem we replace the Dirichlet condition at $x=1$ with the Sommerfeld radiation condition, which in one dimension is a simple local condition of Robin type:

$$
\begin{aligned}
-u^{\prime \prime}-k^{2} u & =f \quad \text { on }(0,1), \\
u(0) & =0, \\
u^{\prime}(1)-i k u(1) & =0 .
\end{aligned}
$$

For the constant source function $f \equiv 1$ we obtain the exact solution

$$
\begin{aligned}
u(x) & =\frac{1}{2 k^{2}}\left(\left(e^{i k}-2\right) e^{i k x}-e^{i k} e^{-i k x}-2\right) \\
& =\frac{-1-\cos (k x)-\sin k \sin (k x)-i(1-\cos k) \sin (k x)}{k^{2}} .
\end{aligned}
$$

The same example with $f \equiv-1$ was used in [36] but the expression given there contains a sign error.

We apply the modifications of the basic multigrid V-cycle as developed in the previous sections to the two model problems using the same uniform second-order finite difference discretization considered before. In all cases, the coarse-grid operators were formed not by Galerkin projection but by using the same finite difference scheme on the coarse grid as on the fine grid. Specifically, we use two-step Jacobi smoothing with the parameters chosen depending on the relation of the discrete eigenvalues of the Dirichlet problem as developed in Section 2.3. In all the estimates of the required number of smoothing steps we set the parameter $\epsilon$ indicating the desired reduction factor to 0.1 . Moreover, we apply the modified wave number as given in (3.16) on the coarse levels. To illustrate the behavior of the modified multigrid scheme we apply it to the model problems over the discrete range of wave numbers given in (4.3).

\subsection{Two-Grid Experiments}

We begin with two-grid iterations for the model problem (5.1) with a coarse-grid correction employing a modified wave number on the coarse grid according to (3.16) combined with two-step Jacobi smoothing with the smoothing parameters chosen according to the location of the eigenvalues of the discrete Dirichlet-Helmholtz operator 

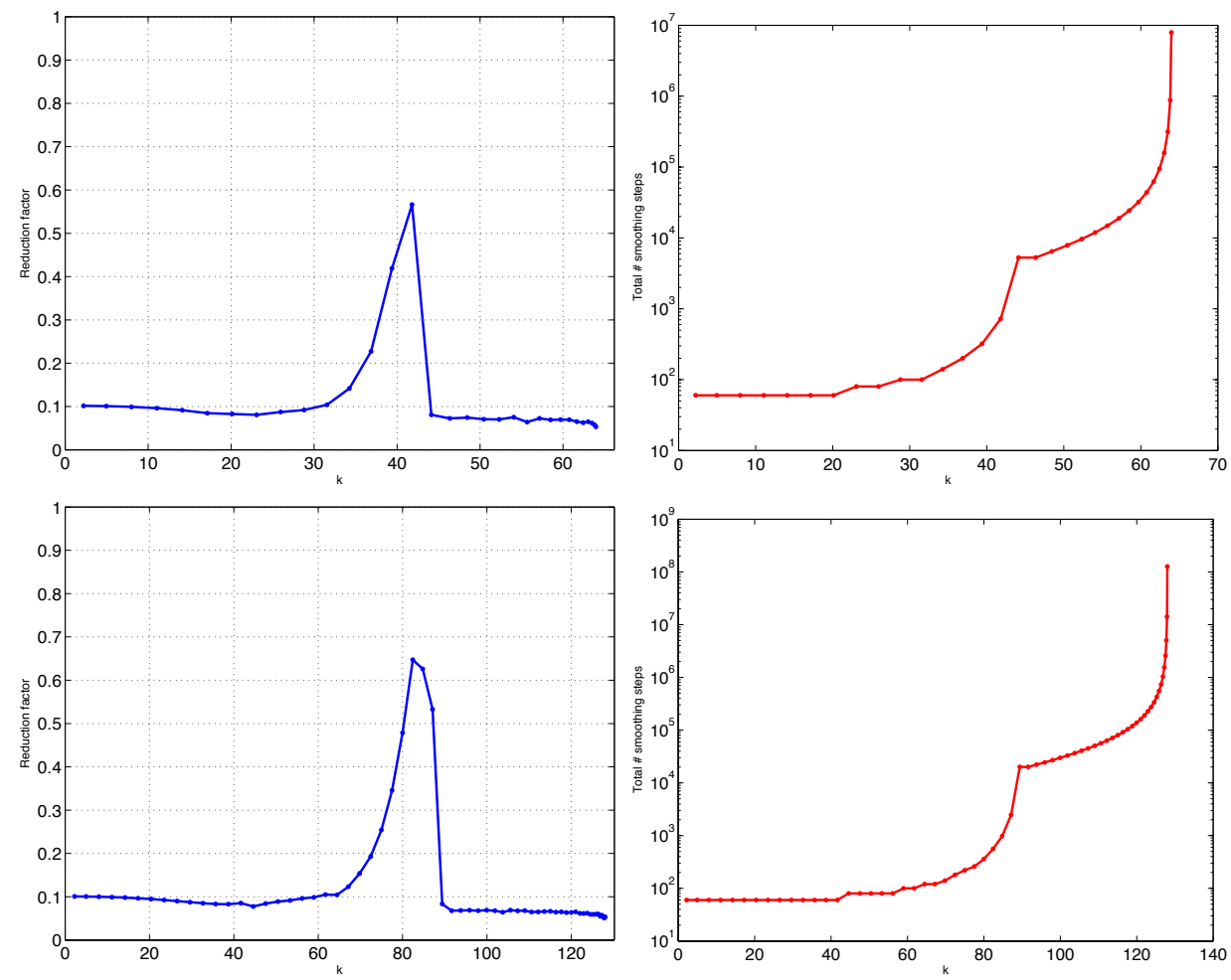

Figure 23. Measured error reduction factor for a two-grid method (left) and total number of smoothing steps (right) using two-step Jacobi smoothing and modified wave number for problem (5.1) with mesh width $h=1 / 32$ (top) and $h=1 / 64$ (bottom) for the sequence (4.3) of wave numbers $k$.

as described in Section 2.3. Following the observations made in Section 4, we employ a modified wave number on all coarse grids which still have a resolution of at least $\lambda / h \geq 5$. Figure 23 shows the corresponding measured reduction factors and total number of smoothing iterations. We note the region just below $k h=\sqrt{2}$ where a reduction factor much larger than the desired $\epsilon=0.1$ results. As discussed in Section 4.2, this is due to the weakening of the effect of shifting the wave number on the coarse grid, which was not taken into account when estimating the number of smoothing steps required based on the smoother alone. When using the remedy proposed in Section 4.2, which means we switch for the estimate of the number of smoothing steps in the two-step Jacobi smoother from the first to the second case already when $k h \geq \sqrt{2}-0.3$, leads to the results shown in Figure 24. We observe that the two grid cycle has now a uniform error reduction factor of about 0.2 .

We also notice, however, that the number of smoothing steps required by the estimate (2.18) on the resonance level is excessively high. We therefore replace the 2-step 

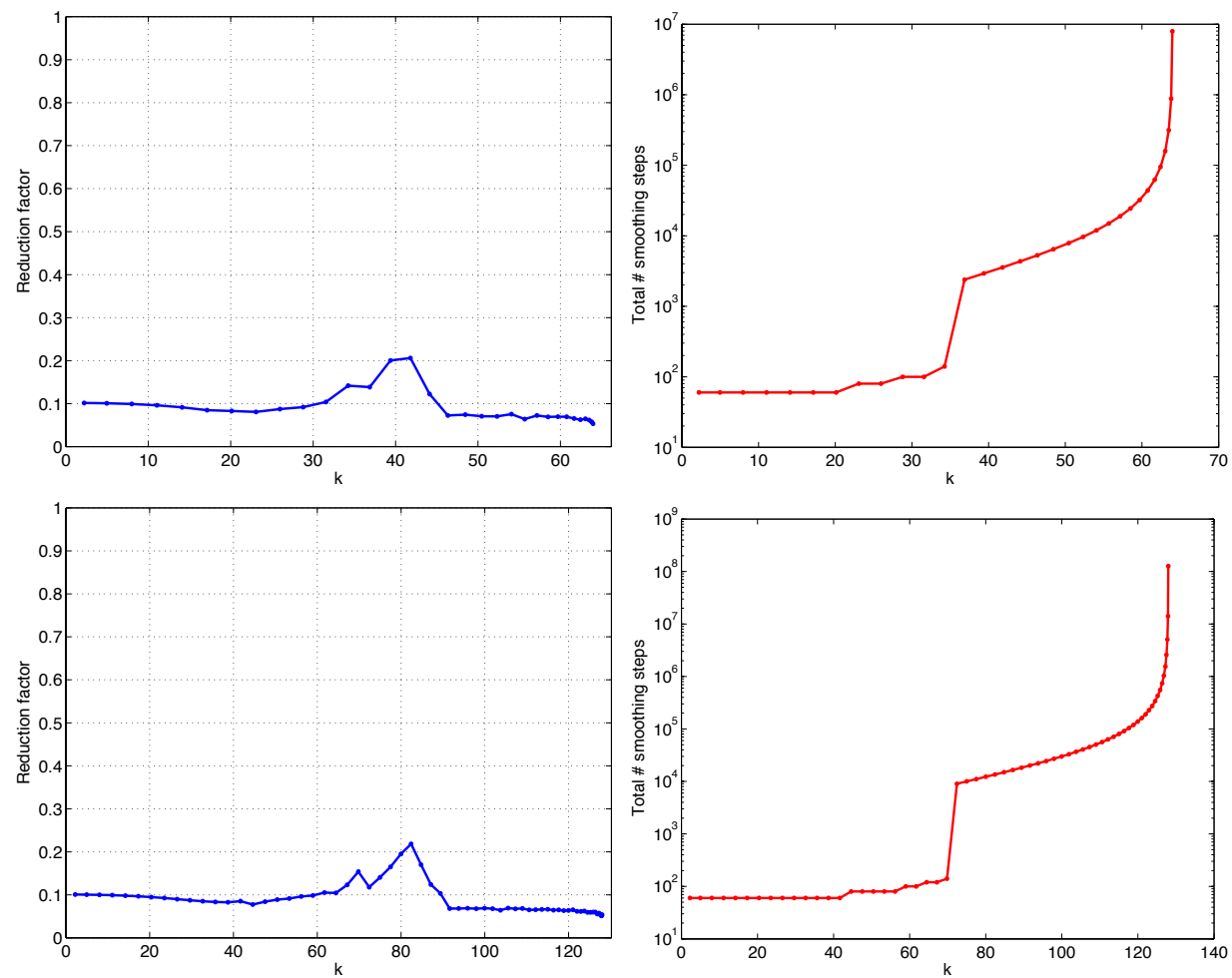

Figure 24. Reduction factor of the two-grid method using two-step Jacobi with early transition to resonance level number of smoothing steps (2.18) and modified wave number for problem (5.1) with mesh width $h=1 / 32$ (top) and $h=1 / 64$ (bottom).

Jacobi smoother now by GMRES whenever we would have used the estimate (2.18) for the number of smoothing steps, i.e., on the resonance level and slightly before, $\sqrt{2}-0.3<k h<2$, and we use $\nu=\frac{3 k}{2 \pi}$ as the number of GMRES smoothing steps, which is linear in $k$. This leads to the reduction factors and total smoothing counts shown in Figure 25 for $h=1 / 32$ and $h=1 / 64$. We observe again a uniformly small reduction factor across all wave numbers and a much smaller number of smoothing steps.

\subsection{Multi-grid Experiments, Complexity}

We next show multigrid experiments, beginning with a complexity estimate. For the Laplacian, if we perform a fixed number $\nu$ of smoothing steps, and the smoother has a 

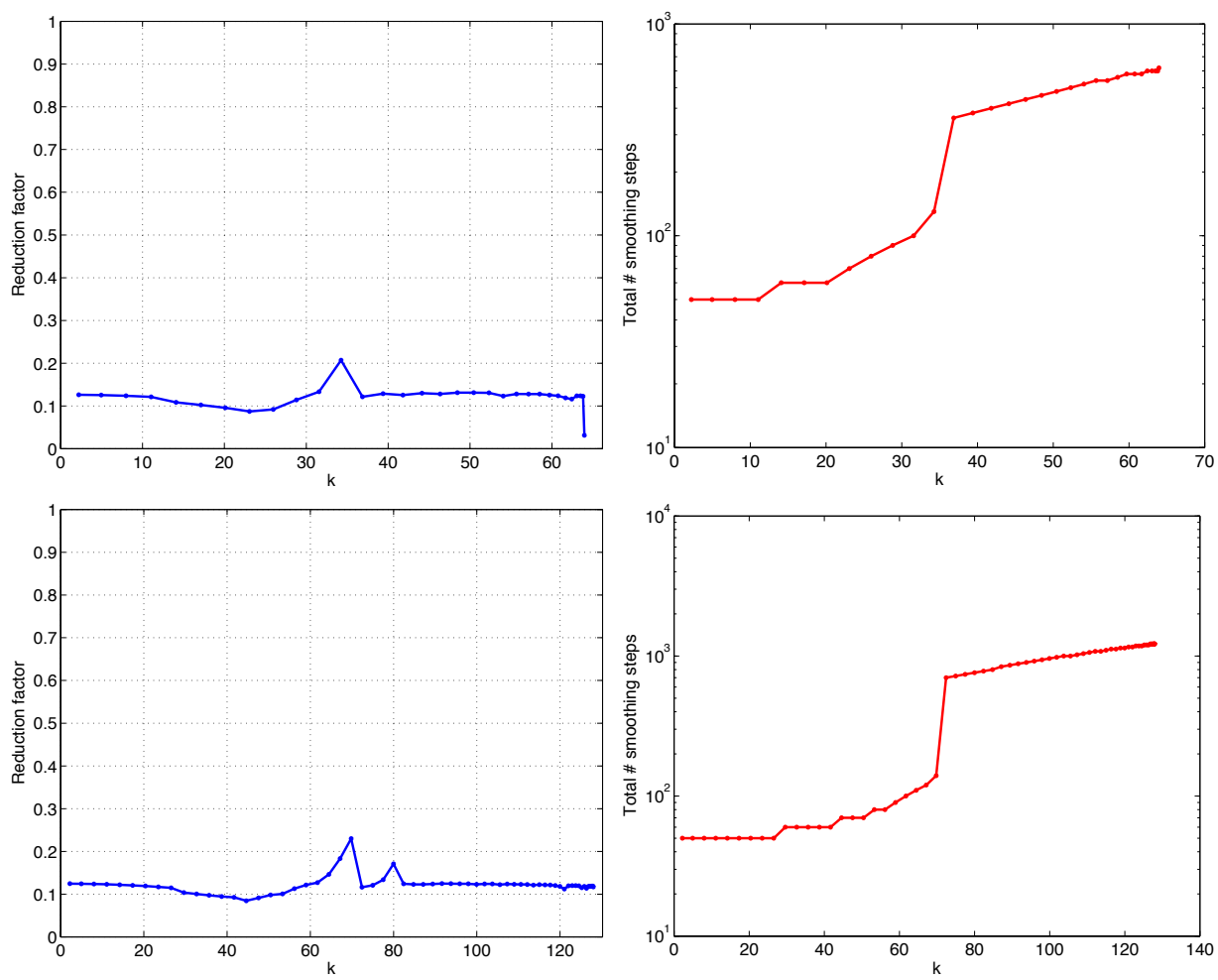

Figure 25. Reduction factor of the two-grid method using two-step Jacobi with early transition to resonance level with Krylov smoothing and modified wave number for problem (5.1) with mesh width $h=1 / 32$ (top) and $h=1 / 64$ (bottom).

cost per step linear in $\frac{1}{h}$, e.g., $\frac{C_{J}}{h}$, then the cost of one V-cycle is, for $h=2^{-m}$,

$$
\begin{aligned}
c_{\Delta} & :=\nu \frac{C_{J}}{h}+\nu \frac{C_{J}}{2 h}+\ldots+\nu C_{J}=\nu \frac{C_{J}}{h} \sum_{j=0}^{m} \frac{1}{2^{j}}=\nu \frac{C_{J}}{h}\left(2-\frac{1}{2^{m}}\right) \\
& =\nu \frac{C_{J}}{h}(2-h)<\nu \frac{2 C_{J}}{h} .
\end{aligned}
$$

In the Helmholtz case, we have seen that the number of smoothing steps becomes large on one particular level, the resonance level, where $O\left(k^{2}\right)$ two-step Jacobi smoothing steps, or $O(k)$ Krylov smoothing steps are necessary. On the remaining levels the number of smoothing steps remains again bounded by a number $\nu=O(1)$. The cost of our multigrid algorithm therefore contains a component like for the Laplacian, $c_{\Delta}$, and the cost of the resonance level. If we assume that, to avoid the pollution effect, we have chosen a fine-level resolution satisfying $k^{3} h^{2}=C$, then we have in the multigrid 
hierarchy, before reaching the resonance level $\ell$, further levels, where $\ell$ satisfies

$$
k\left(2^{\ell} h\right)<\sqrt{2}, \quad 2^{\ell} \approx \frac{\sqrt{2}}{k h},
$$

see (2.12). The size of the system on the resonance level is then $\frac{1}{2^{\ell+1} h}=\frac{k}{2^{3 / 2}}$, where we used estimate (5.3). On this level, we need either $O\left(k^{2}\right)$ two-step Jacobi smoothing steps, which leads to a cost of

$$
c_{J}=k^{2} C_{J} \frac{k}{2^{\frac{3}{2}}}=\frac{C_{J} k^{3}}{2^{\frac{3}{2}}}=\frac{C_{J} C}{2^{\frac{3}{2}} h^{2}},
$$

where we used $k^{3} h^{2}=C$, or $O(k)$ Krylov smoothing steps, which, assuming that also the cost of Krylov steps is linear in the size of the system with constant of proportionality $C_{K}$, leads to a cost

$$
c_{K}=k C_{k} \frac{k}{2^{\frac{3}{2}}}=\frac{C_{K} k^{2}}{2^{\frac{3}{2}}}=\frac{C_{K} C}{2^{\frac{3}{2}} h^{\frac{4}{3}}} .
$$

The overall cost of this multigrid algorithm is therefore, for one V-cycle, bounded by

$$
c_{J}^{t o t}<c_{\Delta}+c_{J}<\nu \frac{2 C_{J}}{h}+\frac{C_{J} C}{2^{\frac{3}{2}} h^{2}}
$$

in the case of the 2-step Jacobi smoother on the resonance level, and

$$
c_{K}^{t o t}<c_{\Delta}+c_{K}<\nu \frac{2 C_{J}}{h}+\frac{C_{K} C}{2^{\frac{3}{2}} h^{\frac{4}{3}}}
$$

in the case where on one level, the resonance level, one uses a Krylov smoother with $O(k)$ steps, and otherwise 2-step Jacobi. We see that, compared to the case of the Laplacian, each V-cycle has more than linear complexity with respect to the dimension of the finest system, $1 / h$ : with Jacobi on the resonance level, one pays $O\left(h^{-2}\right)$ to have a fixed iteration number independent of the mesh size $h$, and hence $k$, and with Krylov smoothing on the resonance level one pays $O\left(h^{-4 / 3}\right)$ per V-cycle to have a fixed iteration number independent of $h$ and $k$. This latter result seems very much acceptable, however, for the difficult case of the Helmholtz equation: one pays $O\left(h^{-4 / 3}\right)$ instead of $O\left(h^{-1}\right)$ per V-cycle, for iteration numbers independent of $h$ and $k$.

We now show numerical experiments for multigrid V-cycles over six levels applied to the Helmholtz equation using the modifications described in this chapter. We start by showing error reduction rates in Figure 26. On the left, we see that, for the problem (5.1) with pure Dirichlet boundary conditions, for which our analysis of the two-grid cycle was performed, the addition of further grid levels still gives uniform error reduction for all wave numbers $k$, by a factor bounded by about 0.3 . 

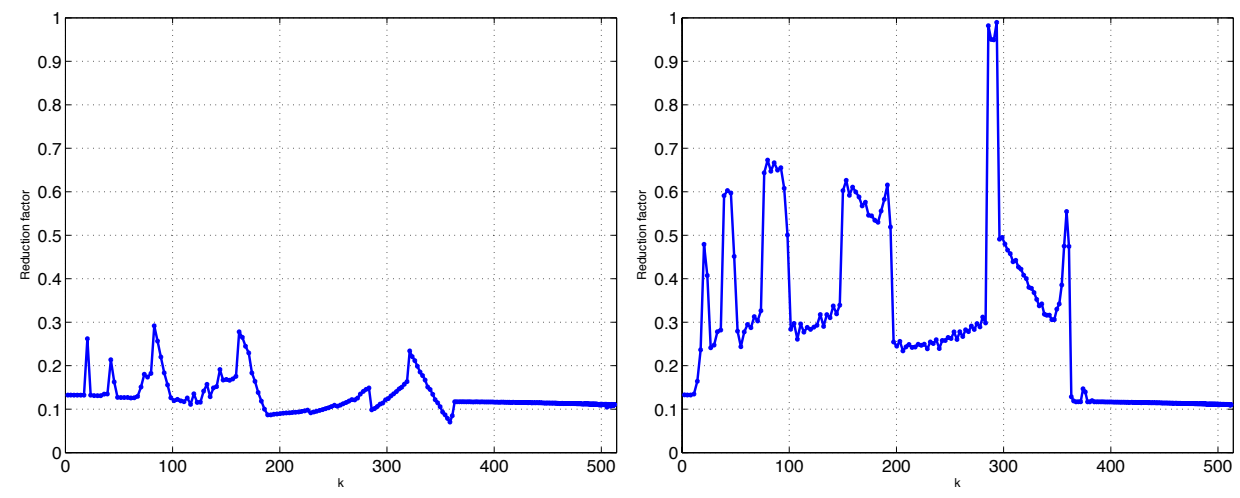

Figure 26. V-cycle with 6 levels using 2-step Jacobi smoothing and Krylov smoothing on the resonance level and modified wave number for problem (5.1) (left) and problem (5.2) (right) with mesh width $h=1 / 256$.

On the right in Figure 26, we also show a numerical experiment for a situation not covered by our analysis, namely problem (5.2) which features a Sommerfeld radiation condition at one boundary. We have observed that the two-step Jacobi smoother, whose parameters were tuned to the Dirichlet case in Section 2.3, are not optimal for this case and that the modified wave number had to be restricted to grids for which $k h<2.3$, a somewhat better resolution than $\lambda / h=5$, to obtain a convergent scheme. Extending the analysis to this situation is more difficult as the discrete operator is no longer normal and, therefore, pure eigenvalue analysis of the smoother is not sufficient. Nevertheless, using the shift of the wavenumber and smoothing techniques developed for the Dirichlet problem, we onetheless obtain a convergent multigrid V-cycle across the full range of wave numbers. Combining this type of solver with an (outer) Krylov acceleration could lead to an effective solver.

We finally show a sequence of realistic numerical experiments, starting on a fine grid with enough resolution to give an accurate discrete approximation, which means to use a scaling of $k$ and $h$ that bounds the pollution error of our centered finite-difference discretization scheme. As shown in $[35,36,3]$, this requires that $k^{3} h^{2}$ remain bounded as the wave number is increased and the fine mesh refined.

We begin with a mesh width of $h=1 / 64$ and $k=40$, yielding a resolution of about 10 points per wavelength. We then choose the wave numbers on the sequence of successively halved meshes with $h=1 / 128, \ldots, h=1 / 2048$ so that $k^{3} h^{2}$ has about the same value for all problems. We show measured reduction rates for two variants of our multigrid method employing V-cycles containing two through six grids levels for the 1D model problem (5.1) in Table 3 and the Sommerfeld problem (5.2) in Table 4. The first variant employs $O\left(k^{2}\right)$ two-step Jacobi smoothing steps and the second $O(k)$ Krylov smoothing steps on the resonance level using GMRES. Spanning a range of wave numbers $k$ from 63.74 to 403.04, which corresponds to roughly 12 


\begin{tabular}{|c|ccccc|}
\hline$h$ & $1 / 128$ & $1 / 256$ & $1 / 512$ & $1 / 1024$ & $1 / 2048$ \\
$k$ & 63.74 & 101.43 & 161.12 & 255.38 & 403.04 \\
\hline \multicolumn{5}{|c|}{ two-step Jacobi smoothing on resonance level } \\
\hline 2-grid & 0.0891 & 0.1147 & 0.1111 & 0.1605 & 0.1448 \\
3-grid & 0.1177 & 0.1216 & 0.1291 & 0.1686 & 0.1579 \\
4-grid & 0.1091 & 0.1291 & 0.3157 & 0.2146 & 0.1604 \\
5-grid & 0.1091 & 0.1291 & 0.3157 & 0.1649 & 0.1679 \\
6-grid & 0.1091 & 0.1291 & 0.3157 & 0.1649 & 0.1679 \\
\hline \multicolumn{7}{|c|}{ GMRES smoothing 00 resonance level } \\
\hline 2-grid & 0.0891 & 0.1147 & 0.1111 & 0.1605 & 0.1448 \\
3-grid & 0.1177 & 0.1216 & 0.1291 & 0.1686 & 0.1580 \\
4-grid & 0.1107 & 0.1187 & 0.3157 & 0.2146 & 0.1604 \\
5-grid & 0.1107 & 0.1195 & 0.3157 & 0.2086 & 0.1686 \\
6-grid & 0.1107 & 0.1195 & 0.3157 & 0.2086 & 0.1686 \\
\hline
\end{tabular}

Table 3. Measured reduction rates for two multigrid variants on a sequence of problems of type (5.1) with $k^{2} h^{3}$ held constant to bound pollution error. The smoothing on the resonance level employs $O\left(k^{2}\right)$ steps for two-step Jacobi and $O(k)$ for GMRES smoothing.

and 64 full waves across the domain $\Omega=(0,1)$, respectively, we obtain in the case of Dirichlet boundary conditions reduction rates around 0.1 for the two-grid method to at worst 0.32 for the six-grid method. For Problem (5.2) with the Sommerfeld boundary condition, we obtain for the two-grid method again reduction rates of about 0.1 , and at worst 0.62 for the six-grid method. In all our results, while the error reduction rates can deteriorate a bit when the resonance level becomes part of the multigrid hierarchy, they become stable as one increases the number of levels further, which shows that the methods are now clearly independent of the number of levels used, crossing the resonance level in a stable fashion. We note that we have obtained even somewhat better rates when standard Jacobi smoothing is used in place of our two-step Jacobi smoother on sufficiently fine grids.

\section{Conclusions}

We have explained in this chapter in detail for a 1D model problem why one cannot simply use a standard multigrid method to solve discretized Helmholtz problems. Both the smoother and the coarse grid correction will fail in that case. Standard smoothers amplify low frequency error components, and are thus not stable, a fact that is well known. The coarse grid correction fails because of the incorrect dispersion relation 


\begin{tabular}{|c|ccccc|}
\hline$h$ & $1 / 128$ & $1 / 256$ & $1 / 512$ & $1 / 1024$ & $1 / 2048$ \\
$k$ & 63.74 & 101.43 & 161.12 & 255.38 & 403.04 \\
\hline \multicolumn{5}{|c|}{ two-step Jacobi smoothing on resonance level } \\
\hline 2-grid & 0.0936 & 0.1249 & 0.1442 & 0.1656 & 0.1618 \\
3-grid & 0.2884 & 0.2187 & 0.1794 & 0.1899 & 0.1916 \\
4-grid & 0.2864 & 0.3926 & 0.6222 & 0.2714 & 0.2370 \\
5-grid & 0.2864 & 0.3963 & 0.6222 & 0.2761 & 0.4117 \\
6-grid & 0.2864 & 0.3963 & 0.6222 & 0.2761 & 0.4117 \\
\hline \multicolumn{7}{|c|}{ GMRES smoothing on resonance level } \\
\hline 2-grid & 0.0936 & 0.1249 & 0.1442 & 0.1656 & 0.1618 \\
3-grid & 0.2884 & 0.2187 & 0.1794 & 0.1899 & 0.1916 \\
4-grid & 0.3077 & 0.2837 & 0.6222 & 0.2714 & 0.2370 \\
5-grid & 0.3077 & 0.2837 & 0.6222 & 0.2838 & 0.2893 \\
6-grid & 0.3077 & 0.2838 & 0.6222 & 0.2838 & 0.2893 \\
\hline
\end{tabular}

Table 4. Measured reduction rates for two multigrid variants on a sequence of problems of type (5.2) with $k^{2} h^{3}$ held constant to bound pollution error. The smoothing on the resonance level employs $O\left(k^{2}\right)$ steps for two-step Jacobi and $O(k)$ for GMRES smoothing.

(phase error) on coarser and coarser grids, a fact that is less well known.

We then designed a two-step Jacobi smoother which is convergent for all levels, and this required scheduling specific numbers of smoothing steps depending on the wave number $k$ and the mesh size $h$ on the current level, in order to obtain uniform error reduction in the high frequency modes. On one particular level, which we call the resonance level, an exceedingly high number of smoothing steps is needed, namely $O\left(k^{2}\right)$ steps, if one insists on uniform error reduction in the high-frequency components on that level. A better approach is to choose a more suitable higher degree polynomial smoother on the resonance level, and we used GMRES in this case, and then "only" $O(k)$ smoothing steps are needed for a uniform error reduction in the high-frequency components.

In order to alleviate the dispersion relation problem on coarser meshes, we introduced a shift in the wave number, adapted to the mesh size $h$ of the level. In one spatial dimension, we can obtain uniform error reduction with the two and multigrid cycle using this technique, and we showed that the overall complexity of our multigrid algorithm is $O\left(h^{-4 / 3}\right)$ when we use on the resonance level a Krylov smoother, compared to the optimal complexity of multigrid for the Laplacian alone, which is $O\left(h^{-1}\right)$.

We illustrated our results with numerical experiments in one spatial dimension, 
which illustrates well that a fixed number of iterations suffices to solve Helmholtz equations in one dimension with this adapted multigrid method, still only using standard, but adapted components, independently of the wave number $k$ and the mesh size $h$.

In higher dimensions, our smoothing analysis still applies, and the two-step Jacobi smoother will work well, as will the Krylov smoother on the resonance level. A perfect dispersion relation correction, however, will not be possible any more with only a shift of the wave number on coarser and coarser levels, since the dispersion relation is now a curve in two dimensions, or a surface in three dimensions. We have, however, observed that higher order discretization schemes lead to nearly spherical dispersion surfaces. We are currently analyzing how much a scalar dispersion correction can still help in higher dimension; these results will appear in a future publication.

\section{Bibliography}

[1] Mark Ainsworth, Discrete Dispersion Relation for $h p$-Version Finite Element Approximation at High Wave Number, SIAM J. Numer. Anal. 42 (2004), 553-575.

[2] Tuomas Airaksinen, Erkki Heikkola, Anssi Pennanen and Jari Toivanen, An algebraic multigrid based shifted-Laplacian preconditioner for the Helmholtz equation, Journal of Computational Physics 226 (2007), 1196-1210.

[3] Ivo M Babuska and Stefan A Sauter, Is the pollution effect of the FEM avoidable for the Helmholtz equation considering high wave numbers?, SIAM review 42 (2000), 451-484.

[4] Randolph E. Bank, A comparison of two multilevel iterative methods for nonsymmetric and indefinite elliptic finite element equations, 18 (1981), 724-743.

[5] A. Bayliss, C.I. Goldstein and E. Turkel, An iterative method for the Helmholtz equation, J. Comput. Phys. 49 (1983), 443-457.

[6] Alvin Bayliss, Charles I. Goldstein and Eli Turkel, The numerical solution of the Helmholtz equation for wave propagation problems in underwater acoustics, 11 (1985), 655-665.

[7] Folkmar A. Bornemann and Rolf Krause, Classical and Cascadic Multigrid-A Methodological Comparison, in: Ninth International Conference on Domain Decomposition Methods (Petter E. Bjørstadt, Magne E. Espedal and David E. Keyes, eds.), pp. 64-71, 1998.

[8] James H. Bramble, Joseph E. Pasciak and Jinchao Xu, The Analysis of Multigrid Algorithms for Nonsymmetric and Indefinite Elliptic Problems, 51 (1988), 389-414.

[9] A. Brandt and S. Ta'asan, Multigrid Methods for Nearly Singular and Slightly Indefinite Problems, in: Multigrid Methods II, pp. 99-121, Springer, 1986.

[10] Achi Brandt and Oren E. Livne, Multigrid Techniques, 1984 Guide with Applications to Fluid Dynamics, Revised Edition, Classics in Applied Mathematics 67, SIAM, 2011.

[11] Achi Brandt and Irene Livshits, Wave-ray multigrid method for standing wave equations, Electron. Trans. Numer. Anal. 6 (1997), 162-181. 
[12] William L. Briggs, Van Emden Henson and Steve F. McCormick, A Multigrid Tutorial, second ed, SIAM, 2000.

[13] Gary C. Cohen, Higher-Order Numerical Methods for Transient Wave Equations, Scientific Computation, Springer, Berlin, Heidelberg, 2002.

[14] David L. Colton and Rainer Kress, Integral Equation Methods in Scattering Theory, Wiley, New York, 1983.

[15] Siegfried Cools and Wim Vanroose, Local Fourier Analysis of the Complex Shifted Laplacian preconditioner for Helmholtz problems, arXiv preprint arXiv:1112.5346 (2011).

[16] B. Després, Méthodes de décomposition de demains pour les problèms de propagation d'ondes en régime harmonique, Ph.D. thesis, Université Paris IX Dauphine, 1991.

[17] Howard C. Elman, Oliver G. Ernst and Dianne P. O'Leary, A Multigrid Method Enhanced by Krylov Subspace Iteration for Discrete Helmholtz Equations, SIAM J. Sci. Comp. 23 (2001), 1290-1314.

[18] Björn Engquist and Lexing Ying, Sweeping preconditioner for the Helmholtz equation: hierarchical matrix representation, Communications on pure and applied mathematics 64 (2011), 697-735.

[19] __ Sweeping preconditioner for the Helmholtz equation: moving perfectly matched layers, Multiscale Modeling \& Simulation 9 (2011), 686-710.

[20] Y.A. Erlangga, Advances in Iterative Methods and Preconditioners for the Helmholtz Equation, Archives Comput. Methods in Engin. 15 (2008), 37-66.

[21] Y.A. Erlangga, C. Vuik and C.W. Oosterlee, On a class of preconditioners for solving the Helmholtz equation, Applied Numerical Mathematics 50 (2004), 409-425.

[22] O.G. Ernst and M.J. Gander, Why it is Difficult to Solve Helmholtz Problems with Classical Iterative Methods, Numerical Analysis of Multiscale Problems (I. Graham, T. Hou, O. Lakkis and R. Scheichl, eds.), Springer Verlag, 2012, pp. 325-363.

[23] C. Farhat, P. Avery, R. Tezaur and J. Li, FETI-DPH: a dual-primal domain decomposition method for accoustic scattering, Journal of Computational Acoustics 13 (2005), 499-524.

[24] Charbel Farhat, Philip Avery, Radek Tesaur and Jing Li, FETI-DPH: a dual-primal domain decomposition method for acoustic scattering, Journal of Computational Acoustics 13 (2005), 499-524.

[25] Charbel Farhat, Antonini Macedo and Radek Tezaur, FETI-H: a Scalable Domain Decomposition Method for High Frequency Exterior Helmholtz Problem, in: Eleventh International Conference on Domain Decomposition Method (Choi-Hong Lai, Petter Bjørstad, Mark Cross and Olof Widlund, eds.), pp. 231-241, DDM.ORG, 1999.

[26] M. J. Gander and F. Nataf, AILU: A preconditioner based on the analytic factorization of the elliptic operator, Num. Lin. Alg. and Appl. 7 (2000), 543-567.

[27] M. J. Gander and H. Zhang, Domain decomposition methods for the Helmholtz equation: a numerical investigation., in: Domain Decomposition Methods in Science and Engineering XX, Lecture Notes in Computational Science and Engineering (R. Bank, M. Holst and J. Xu, eds.), San Diego, p. to appear, Springer-Verlag, 2012. 
[28] Martin J. Gander, Laurence Halpern and Frédéric Magoulès, An Optimized Schwarz Method with Two-Sided Robin Transmission Conditions for the Helmholtz Equation, Int. J. for Num. Meth. in Fluids 55 (2007), 163-175.

[29] Martin J. Gander, Frédéric Magoulès and Frédéric Nataf, Optimized Schwarz Methods without Overlap for the Helmholtz Equation, SIAM J. Sci. Comput. 24 (2002), 38-60.

[30] Martin J. Gander and Frédéric Nataf, An Incomplete LU Preconditioner for Problems in Acoustics, Journal of Computational Acoustics 13 (2005), 1-22.

[31] M.J. Gander, Ivan G. Graham and Euan A. Spence, Analysis of the Shifted Laplace Preconditioner for the Helmholtz Equation, in preparation.

[32] M.B. Van Gijzen, Y.A. Erlangga and C. Vuik, Spectral analysis of the discrete Helmholtz operator preconditioned with a shifted Laplacian, SIAM J. Sci. Comput. 29 (2007), 1942 1958.

[33] Wolfgang Hackbusch, Multi-Grid Methods and Applications, Springer-Verlag, 1985.

[34] G. Ronald Hadley, A complex Jacobi iterative method for the indefinite Helmholtz equation, Journal of Computational Physics 203 (2005), 358-370.

[35] Frank Ihlenburg and Ivo Babuška, Finite Element Solution to the Helmholtz Equation with High Wave Number. Part I: The $h$-version of the FEM, Computer Methods in Applied Mechanics and Engineering 39 (1995), 9-37.

[36] _ Finite Element Solution of the Helmholtz Equation with High Wave Number Part II: The $h-p$ Version of The FEM, SIAM J. Numer. Anal. 34 (1997), 315-358.

[37] Seongjai Kim and Soohyun Kim, Multigrid simulation for high-frequency solutions of the Helmholtz problem in heterogeneous media, SIAM Journal on Scientific Computing 24 (2002), 684-701.

[38] B. Lee, T. Manteuffel, S. McCormick and J. Ruge, First-order system least squares (FOSLS) for the Helmholtz equation, SIAM J. Sci. Comp. 21 (2000), 1927-1949.

[39] Irene Livshits, Multigrid Solvers for Wave Equations, Ph.D. thesis, Bar-Ilan University, Ramat-Gan, Israel, 1996.

[40] Irene Livshits and Achi Brandt, Accuracy properties of the wave-ray multigrid algorithm for Helmholtz equations, SIAM Journal on Scientific Computing 28 (2006), 1228-1251.

[41] Lewis Fry Richardson, On the Approximate Arithmetical Solution by Finite Differences of Physical Problems Involving Differential Equations, with an Application to the Stresses in a Masonry Dam, Proceedings of the Royal Society of London. Series A 83 (1910), 335-336.

[42] Yair Shapira, Multigrid Techniques for Highly Indefinite Equations, in: Proceedings 1995 Copper Mountain Conference on Multigrid Methods, 1995, available at http://www.c3.lanl.gov/ yairs/.

[43] Richard Vynne Southwell, Relaxation methods in engineering science : a treatise on approximate computation, Oxford University Press, 1940.

[44] John C. Strikwerda, Finite Difference Schemes and Partial Differential Equations, 2nd ed, SIAM, Philadelphia, 2004. 
[45] Ulrich Trottenberg, C. Cornelis W. Oosterlee and Anton Schüller, Multigrid, Academic Press, 2001.

[46] Petr Vanek, Jan Mandel and Marian Brezina, Two-level algebraic multigrid for the Helmholtz problem, Contemporary Mathematics 218 (1998), 349-356.

[47] Wim Vanroose, Bram Reps and Hisham bin Zubair, A polynomial multigrid smoother for the iterative solution of the heterogeneous Helmholtz problem, arXiv preprint arXiv:1012.5379 (2010).

[48] Wim Vanroose, Bram Reps, Hisham Bin Zubair et al., Multigrid preconditioners based on polynomial smoothers for the Helmholtz equation with absorbing layers, in: International Conference On Preconditioning Techniques For Scientific And Industrial Applications, Preconditioning 2011, 2011.

[49] Richard S. Varga, Matrix Iterative Analysis, Prentice-Hall, Englewood-Cliffs, NJ, 1962.

[50] Harry Yserentant, On the Multilevel Spltting of Finite Element Spaces for Indefinite Elliptic Boundary Value Problems, 23 (1986), 581-595.

\section{Author information}

Oliver G. Ernst, Fakultät Mathematik und Informatik, TU Bergakademie Freiberg, Akademiestr. 6, 09595 Freiberg, Germany.

E-mail: ernst@math.tu-freiberg.de

Martin J. Gander, Section de Mathématiques, Université de Genève, 2-4 rue du Lièvre, CP 64, CH-1211 Genève, Switzerland.

E-mail: Martin.Gander@unige.ch 